\title{
Uncertainty Propagation in Coupled Atmosphere-Wave-Ocean Prediction System: A Study of Hurricane Earl (2010)
}

\author{
GUOTU Li \\ Duke University, Durham, North Carolina \\ Milan CURCIC AND MoHAMEd ISKANDARANI \\ University of Miami, Miami, Florida \\ SHUYI S. CHEN \\ University of Washington, Seattle, Washington \\ OMAR M. KNIO \\ Duke University, Durham, North Carolina
}

(Manuscript received 7 December 2017, in final form 26 October 2018)

\begin{abstract}
This study focuses on understanding the evolution of Hurricane Earl (2010) with respect to random perturbations in the storm's initial strength, size, and asymmetry in wind distribution. We rely on the Unified Wave Interface-Coupled Model (UWIN-CM), a fully coupled atmosphere-wave-ocean system to generate a storm realization ensemble, and use polynomial chaos (PC) expansions to build surrogate models for time evolution of both the maximum wind speed and minimum sea level pressure in Earl. The resulting PC surrogate models provide statistical insights on probability distributions of model responses throughout the simulation time span. Statistical analysis of rapid intensification (RI) suggests that initial perturbations having intensified and counterclockwise-rotated winds are more likely to undergo RI. In addition, for the range of initial conditions considered RI seems mostly sensitive to azimuthally averaged maximum wind speed and asymmetry orientation, rather than storm size and asymmetry magnitude; this is consistent with global sensitivity analysis of PC surrogate models. Finally, we combine initial condition perturbations with a stochastic kinetic energy backscatter scheme (SKEBS) forcing in the UWIN-CM simulations and conclude that the storm tracks are substantially influenced by the SKEBS forcing perturbations, whereas the perturbations in initial conditions alone had only limited impact on the storm-track forecast.
\end{abstract}

\section{Introduction}

Tropical cyclones (TCs) produce extreme wind, rain, large ocean waves, storm surges, and flooding, which result in significant impact on coastal areas (Stone et al. 1997). It is critical, both scientifically and economically (Letson et al. 2007), to better understand dynamics and improve TC forecasts. This study focuses on the impacts of initial condition uncertainties on the storm track and

\footnotetext{
${ }^{a}$ Current affiliation: King Abdullah University of Science and Technology, Thuwal, Saudi Arabia.
}

Corresponding author: Omar M. Knio,omar.knio@kaust.edu.sa the characteristics of the maximum wind speed (MWS), minimum sea level pressure (MSLP), and likelihood of rapid intensification (RI). The study also attempts to quantify the impacts of stochastic forcing on the storm track, MWS, and MSLP in the absence of, or in combination with initial condition uncertainties.

Numerous studies and efforts have been devoted to improving our current knowledge of TC dynamics, via examining various ocean and atmosphere states in TC events, including ocean surface waves (Walsh et al. 2002; Chen et al. 2013; Chen and Curcic 2016; Curcic et al. 2016), sea surface temperature (Anthes and Chang 1978; Dickey et al. 1998; Latif et al. 2007; Lee and Chen 2014), rain (Senn and Hiser 1959; Rosenfeld et al. 2007), and 
storm surge (Hsu et al. 1997; Hsu 2013). Some researchers use surface wave forecast to estimate storm impact (Alves et al. 2015). Others have conducted hindcast studies with coupled hydrodynamics and wave models on Hurricane Floyd (1999) (Funakoshi et al. 2008) and Hurricane Ike (2008) (Hope et al. 2013) and suggested significant impact of wind-induced waves on storm tides. Donelan et al. (2012) developed a surface wave model that showed plausible prediction results for both Hurricane Bonnie (1998) and Hurricane Ike (2008). Advanced fully coupled atmosphere-wave-ocean modeling of Hurricane Ivan (2004) (Smith et al. 2013) and Hurricane Frances (2004) (Chen et al. 2007, 2013) suggested significant influence of wind-wave coupling and ocean-wave currents coupling on the atmosphereocean interaction and hurricane response in the two cases, respectively.

Nevertheless, the predictability of TCs and understanding their response to uncertain inputs and forcing remain a major challenge. The sources of these uncertainties can be broadly categorized as structural uncertainties caused by the abstraction of unknown physical laws into the mathematical equations governing the evolution of atmospheric flows; uncertainties in the forecast model input data (such as imperfectly specifying initial conditions, boundary conditions, empirical constants in parameterizations), and uncertainties caused by model errors such as those caused by discretization, numerical errors, missing and/or misparameterized subgridscale processes (e.g., turbulent mixing and scale-dependent convective parameterizations). Additional error sources arise when observational data are assimilated into a forecast. These observation errors have two components: the measurement (or instrument) error and the representation error (the discrepancy between the modeled and observed fields); the representation error can further be decomposed into components resulting from unresolved scales and processes, forward model or observation-operator errors and preprocessing or qualitycontrol errors (Janjić et al. 2018). The present article focuses primarily on quantifying uncertainties on a subset of the model input data, namely the ones relevant to the storm initial configuration, using a response-surface paradigm, and secondarily on model error using a stochastic forcing paradigm; additionally, observational errors will be relevant in the context of comparing ensemble data to observational data.

Initial TC characteristics such as vortex intensity, size, and asymmetry are examples of uncertain model inputs, which are often poorly observed and poorly resolved in standard weather forecasts; numerous data assimilation and vortex specification techniques have been proposed and evaluated for their representation (e.g., Hendricks et al. 2013; Davidson et al. 2014; Zou et al. 2015; Singh et al. 2016). Efforts have also been made to understand the stochastic impacts of uncertainties stemming from the distribution of atmosphere moisture and mesoscale convective system on TC forecasts (Zhang and Sippel 2009; Sippel and Zhang 2010). Subsequently, the intrinsic predictability of TC winds was examined by using idealized simulations of a TC in statistical equilibrium (Brown and Hakim 2013; Hakim 2013). More recently, predictability studies of TC intensity using stochastic kinetic energy backscatter scheme (SKEBS) perturbations were conducted, focusing on 1) the impact of stochastic perturbations at different scales, as well as perturbations in lateral boundary condition on TC intensity predictability (Judt et al. 2016a) and 2) the predictability of RI of a TC (Judt and Chen 2016). However, most predictability studies introduce model uncertainties via nonparametric, global or regional, stochastic perturbations. Whereas stochastic forcing may readily provide information regarding predictability, namely based on the spread of TC simulation ensembles, the insight thus obtained may be limited, because of difficulties in decomposing uncertain inputs into interpretable components and/or the lack of functional relationship between stochastic inputs and model outputs.

To mitigate the difficulty in associating model responses with individual uncertainty sources, we propose in this study a new parametric design in specifying the storm's uncertain initial conditions, which enables us to examine the impact of each individual characteristic of the initial storm on the storm evolution. Specifically, this study quantifies the response of Hurricane Earl (2010) forecasts to uncertainties in storm's initial strength, size, and asymmetry, while allowing the ambient environment (e.g., atmosphere outside the storm, ocean and surface wave fields) to freely evolve in response to the initial vortex perturbations. To this end, we use the fully coupled atmosphere-wave-ocean model, namely the Unified Wave Interface-Coupled Model (UWIN-CM) framework (Chen et al. 2013; Chen and Curcic 2016; Curcic et al. 2016), to simulate Earl's evolution from 27 August to 3 September 2010. The perturbations in the initial vortex are thus allowed to propagate to surface waves, subsurface currents, ocean temperature, and salinity. The propagation of the initial storm uncertainties over this 7-day period (discussed in section 2) is performed via an ensemble of UWIN-CM simulations that sample the uncertain input space. This ensemble is then used to build polynomial chaos-based surrogates of different model responses, namely the time evolutions of MWS and MSLP. [For an overview of the application of PC methods to circulation models, see e.g., Alexanderian et al. (2012), Mattern et al. (2012), Thacker et al. (2012), 
Winokur et al. (2013), Sraj et al. (2013, 2014), Iskandarani et al. (2016a,b), and Siripatana et al. (2017).] These surrogates are then exploited to estimate hurricane statistics and to quantify the relative impact of individual inputs on model outputs as well as their joint sensitivities. Furthermore, the dependence of RI on the uncertain inputs is analyzed.

The choice of Hurricane Earl (2010) as a case study is motivated by the following considerations. First, Earl was a long-lived Atlantic hurricane that went through RI and reached category 4 intensity on the Saffir-Simpson hurricane wind scale (Cangialosi 2011). Earl had no direct interaction with land like many other TCs did, making the analysis and interpretation of the control and perturbation realizations more straightforward. Second, Earl has been thoroughly examined in past literature, from perspectives of dropsonde (Montgomery et al. 2014), lightning (Stevenson et al. 2014), and Doppler radar (Rogers et al. 2015) observations, numerical modeling (Smith et al. 2017), to predictability (Judt et al. 2016a; Judt and Chen 2016). These past studies make Hurricane Earl an excellent case to test new methodologies for uncertainty quantification, and help put our results into context of what is already known about it.

The high-resolution UWIN-CM framework dynamically couples the atmosphere, wave, and ocean circulation models, leading to computationally demanding model runs that have both large CPU and disk storage requirements. As a result, only a limited number of UWIN-CM 7-day forecasts of a hurricane can be afforded. Nonetheless, in light of recent experiences of $\mathrm{Li}$ et al. (2016), we opted for the coupled system capabilities provided by the UWIN-CM, instead of a cheaper atmosphere-only simulation system. Specifically, Li et al. (2016) observed that when perturbing wind forcing and initial conditions independently, the ocean model can in some situations fail or lead to unrealistic response. Thus, our present reliance on a coupled model is also motivated by our desire to gain experiences with a framework that offers the capability of linking and/or correlating multiple sources of uncertainty.

This paper is organized as follows. Section 2 outlines the setup of the UWIN-CM framework employed in this study, including the parameterization of initial storm uncertainties. Section 3 presents the analysis of the response of Hurricane Earl (2010) to perturbations in the initial storm field. In section $3 a$ we briefly introduce and apply a polynomial chaos (PC) methodology to build functional representations, or surrogates, of the quantities of interest (QoIs) in terms of the random inputs. The surrogates are analyzed in section $3 \mathrm{~b}$ to assess their suitability. They are then exploited in section $3 \mathrm{c}$ to quantify the dependence of the MWS and MSLP on the random inputs, and in section $3 \mathrm{~d}$ to investigate the behavior of RI. Section 4 explores the possibility of combining initial condition uncertainty with the global SKEBS forcing perturbations, and compares the results with those obtained by considering uncertain initial conditions only. Section 5 summarizes the conclusions from this study.

\section{Coupled model and initial condition perturbations}

The forecast system used in this study is the UWINCM. It couples the atmospheric [Weather Research and Forecasting (WRF) Model], wave [the Unified Miami Wave Model (UMWM)], and ocean [Hybrid Coordinate Ocean Model (HYCOM)] models in a single framework that can simulate the physical interactions and exchanges between the three components. The model description can be found in appendix A, which largely follows from Chen and Curcic (2016), with modifications to reflect changes in the configuration used in the present study. The remainder of this section reviews the settings of the control simulations and presents the perturbations imposed on the initial storm conditions.

In this study, WRF is configured with a parent domain at a $12-\mathrm{km}$ horizontal grid resolution, and two stormfollowing nests at 4- and 1.3-km horizontal grid resolution. The two inner nests are configured to follow Hurricane Earl as it propagates across the parent domain. The HYCOM domain covers the region of the WRF outer domain. It is configured with $0.04^{\circ}$ horizontal grid spacing (varying from $\approx 3.8$ to $4.4 \mathrm{~km}$ from north to south of the model domain) and 32 vertical levels. The wave model has the same domain as the WRF parent domain and is defined on a 4-km horizontal resolution grid. The wave energy spectrum is represented by 36 directional bins and 37 frequency bins that range from 0.0313 to $2 \mathrm{~Hz}$ on a logarithmic scale.

\section{a. Initial and lateral boundary conditions}

The WRF Model initial and lateral boundary conditions for the control run are from the National Centers for Environmental Prediction (NCEP) Global Forecast System (GFS) at $0.5^{\circ}$ horizontal resolution, initialized at 0000 UTC 27 August 2010. The WRF lateral boundary conditions are updated 6 hourly. The initial and boundary conditions for the ocean model are provided by the global, data assimilated, $0.08^{\circ}$ horizontal resolution daily HYCOM analysis fields (downloaded from http://hycom.org). The wave model is initialized from a calm state, and is forced entirely through the interactive coupling with the atmosphere and ocean circulation models. 


\section{b. Initial condition perturbations}

Figure 1 (top panel) shows the sea surface wind field of the entire simulation domain at $t=0$ (0000 UTC 27 August 2010) from the UWIN-CM forecast, which will be used as a "control run" in this study. Hurricane Earl (2010) started to evolve within the 450-km radius marked by the black circle. We parameterize only the wind field within the $450-\mathrm{km}$ range (i.e., the wind field inside the black circle) and design random perturbations accordingly. Four characteristics of the storm are considered uncertain - the azimuthally averaged maximum wind speed, radius of maximum wind, and magnitude and azimuth of asymmetry-as further described below.

Let $w(r, \beta)$ be the initial wind field within the $450-\mathrm{km}$ storm boundary (as shown in Fig. 1). By conducting an azimuthal fit, we can decompose the wind field as follows:

$$
\begin{aligned}
w(r, \beta)= & A_{0}(r)+A_{1}(r) \cos \left[\beta-\beta_{1}(r)\right] \\
& +\operatorname{Res}(r, \beta), \quad 0 \leq r \leq 450 \mathrm{~km},
\end{aligned}
$$

where $A_{0}(r)$ and $A_{1}(r)$ are wind amplitudes of wavenumbers 0 and 1 , respectively. The term $\beta_{1}(r)$ is the azimuth of the wavenumber- 1 asymmetry, and $\operatorname{Res}(r, \beta)$ denotes the residual wind field. The wind field is perturbed via the four canonical random variables in Table 1 . The wavenumber 0 and 1 amplitudes are defined as

$$
A_{0,1}^{\max }=\max _{r \leq 300 \mathrm{~km}} A_{0,1}(r)
$$

and the radius of maximum wind (RMW) is given by

$$
\mathrm{RMW}=\underset{r \leq 300 \mathrm{~km}}{\operatorname{argmax}} A_{0}(r) .
$$

Note that we limit the RMW to $300 \mathrm{~km}$ in order to allow smooth flow field transition across the $450-\mathrm{km}$ storm boundary. Figure 2 shows examples of initial fields resulting from univariate parameter perturbations.

By perturbing these parameters, we aim to capture the initial uncertainty in TC intensity, vortex size, as well as the magnitude and azimuth of vortex asymmetry. All four of these vortex characteristics are often either poorly sampled because of scarce observations over the open ocean, or undersampled by coarse-resolution global models (e.g., Davidson et al. 2014; Singh et al. 2016) which are used to initialize regional or hurricane-following models such as UWIN-CM.

We choose the perturbation range of $\pm 20 \%$ for $A_{0}$, $A_{1}$, and RMW based on the uncertainty estimates of the best track data by Landsea and Franklin (2013). They found that the intensity estimates range from about $25 \%$ in tropical storms to $10 \%$ in major hurricanes, in the
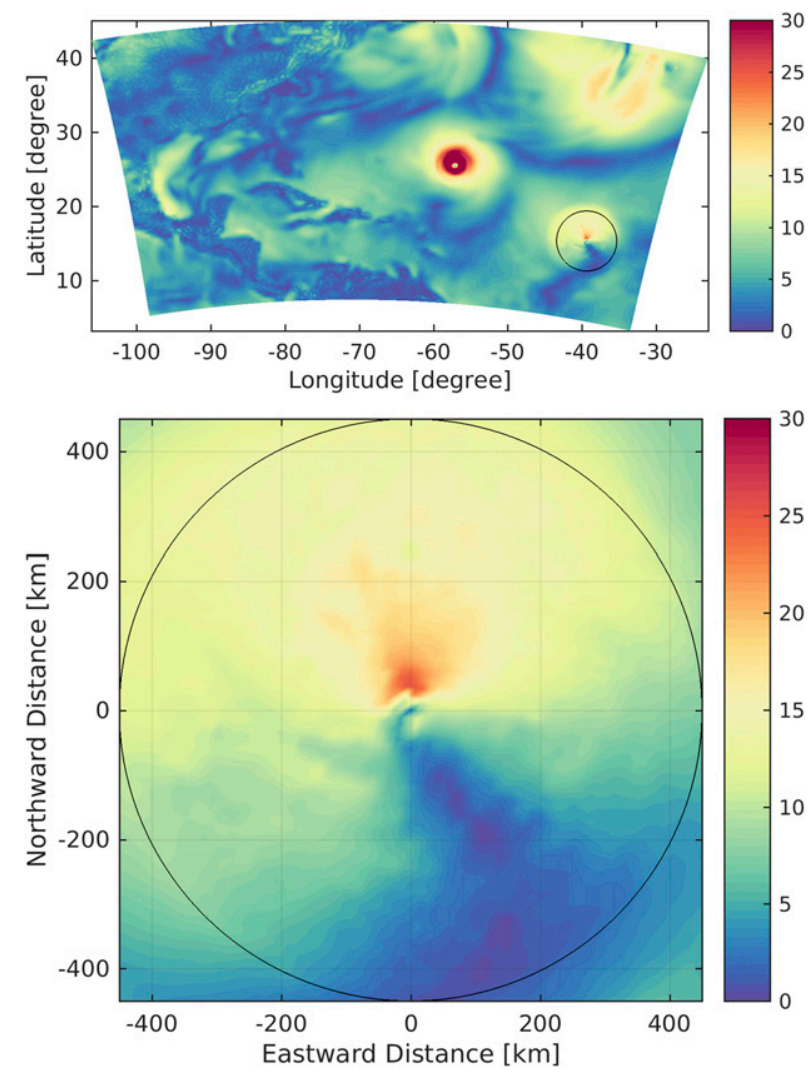

FIG. 1. (top) Sea surface wind field of the entire simulation domain from the control run. Perturbation is applied to the initial wind field within the black circle (radius $=450 \mathrm{~km}$ ). (bottom) Zoom-in plot of the target perturbation area.

absence of aircraft reconnaissance data. When both satellite and aircraft data are available, these uncertainties drop to about $15 \%$ and $8 \%$ in tropical storms and major hurricanes, respectively. The choice of $\pm 20 \%$ for $A_{0}$ and $A_{1}$ is thus in the range of reported uncertainty estimates. Whereas the uncertainty estimates by Landsea and Franklin (2013) are notably higher for the storm size, from about $35 \%$ in tropical storms to about $20 \%$ in major hurricanes, we limit our perturbation range for RMW to $\pm 20 \%$ because too large of a perturbation in storm size made it challenging to produce balanced fields with the storm's environment. All conclusions from this work are thus conditional on the a priori assumptions made about the uncertainty of the selected parameters.

Finally, the motivation for perturbing the vortex asymmetry azimuth comes from wavenumber-1 asymmetry already being embedded in the control simulation, and perturbing only the asymmetry magnitude would not sample the full uncertainty space. For an example, see Xie et al. (2011) who similarly perturbed the vortex asymmetry in an application to storm surge impacts. 
TABLE 1. Uncertain parameters (percentage is relative to the corresponding unperturbed values given by the control run).

\begin{tabular}{cclc}
\hline \hline Parameters & Perturbation range & \multicolumn{1}{c}{ Interpretation } & PC parameters \\
\hline$A_{0}^{\max }$ & {$[-20 \%,+20 \%]$} & Storm strength/intensity & $\xi_{1} \in[-1,1]$ \\
$A_{1}^{\max }$ & {$[-20 \%,+20 \%]$} & Asymmetry strength/intensity & $\xi_{2} \in[-1,1]$ \\
RMW & {$[-20 \%,+20 \%]$} & Radius of max wind & $\xi_{3} \in[-1,1]$ \\
$\theta$ & {$\left[-90^{\circ},+90^{\circ}\right]$} & Storm rotation & $\xi_{4} \in[-1,1]$ \\
\hline
\end{tabular}

More details on perturbation formulation are given in appendix B.

\section{c. Stochastic forcing}

The investigation of the combined effects of input data uncertainties and stochastic forcing required a separate set of UWIN-CM forecast experiments. These experiments relied on the SKEBS approach to perturb the wind and temperature fields over the entire atmospheric model domain throughout the hurricane forecast. The SKEBS configuration used herein is the same as that described in Judt et al. (2016a) and Judt and Chen (2016). Section 4 compares the response of Hurricane Earl to uncertain initial conditions with and without stochastic forcing.

\section{Parametric analysis}

Given the parameterization of initial state of Hurricane Earl (2010) discussed in the previous section, an $N_{r l z}=80$ member ensemble of UWIN-CM simulations is generated via the Latin hypercube sampling (LHS) design (McKay et al. 1979) in the four-dimensional parameter space $\Xi=[-1,1]^{4}$ (we denote this ensemble as $\mathscr{P}_{\text {LHS }}^{80}$ hereafter). For our problem, LHS amounts to partitioning the distributions of each of the (independent) input random variables into intervals of equal probability, and randomly drawing a sample from each interval. A permutation of the individual coordinates is then selected, in such a way that there is no correlation between the inputs. This results in an ensemble of $N_{r l z}$ fourdimensional input vectors, for which UWIN-CM simulations are performed. We choose the maximum 10-m wind speed and MSLP as our primary QoIs. Figures 3 and 4 show model realizations of these QoIs and storm tracks on the sample set $\mathscr{P}_{\text {LHS }}^{80}$, respectively, along with the best estimates of each QoI.

The observed data are provided by the HURDAT2 hurricane database (Landsea and Franklin 2013), produced by the National Hurricane Center (NHC). HURDAT2, commonly known as best track data, is the de facto standard dataset used for baseline verification of most TC simulations in both research and forecasting. Best track data provide estimates of storm center position, MSLP and MWS intensity, and size, every $6 \mathrm{~h}$. They are derived from satellite and aircraft reconnaissance data, when available. Landsea and Franklin (2013) give a detailed account of uncertainty estimates for each parameter in the best track dataset, and these have guided the perturbation range $( \pm 20 \%)$ in our study. Finally, we note the difference in the sampling between best track data and the model fields. Whereas the best track data are based on sparse observations and reported at 6-hourly intervals, the model QoIs are derived from instantaneous fields with full coverage, and output at hourly intervals. Uhlhorn and Nolan (2012) and Nolan et al. (2013) provide detailed uncertainty estimates due to sampling of NHC observations and modeled QoIs.

The realization ensemble of MWS captures the intensity evolution pattern, with a wide envelope enclosing the best estimate by NHC, despite the fact that high and low biases occur at different times. On the other hand, the MSLP ensemble clearly exhibits a high bias in most simulations, and misses the low pressure minimum beyond 31 August when compared with the NHC best estimate. The reintensification of Earl on 2 September is captured to an extent by the simulations in terms of MWS, but not in terms of MSLP. This has an important consequence for the PC analysis, the variability of which is limited by the variability of the coupled model ensemble. Since the control and the coupled model ensemble do not capture the reintensification on 2 September well, the PC analysis is also less representative of the observed storm at that time. Finally, the simulated storm tracks in Fig. 4 show close agreement (slight eastward bias) with the NHC best estimated track, which is an improvement over the current state of the art (Judt et al. 2016a; Smith et al. 2017). The aforementioned storm perturbation mechanism has a relatively small impact on the storm track compared to the SKEBS experiments of Judt and Chen (2016) (see discussion in section 4).

\section{a. PC framework}

The PC approaches have rapidly developed in the past years (Ghanem and Spanos 1991; Le Maître et al. 2001, Le Maitre et al. 2002; Xiu and Karniadakis 2002; Winokur et al. 2013; Conrad and Marzouk 2013; Mycek et al. 2017) and are being widely used for the purpose of uncertainty quantification in ocean general circulation 


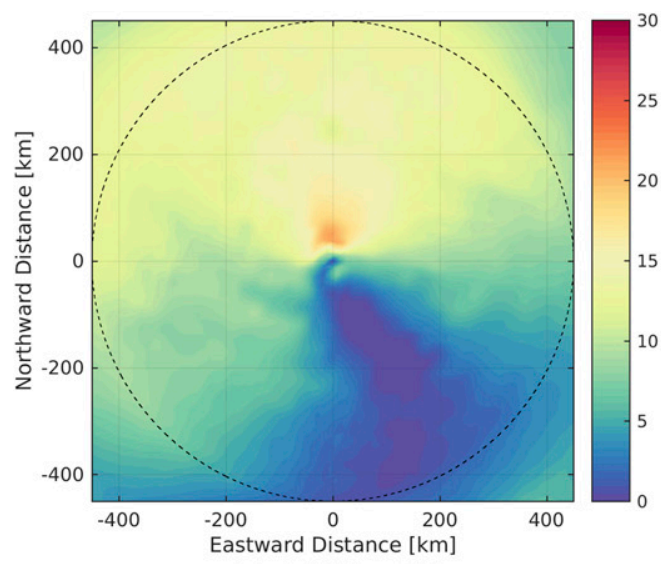

(a) $\Delta A_{0}^{\max }:-20 \%$

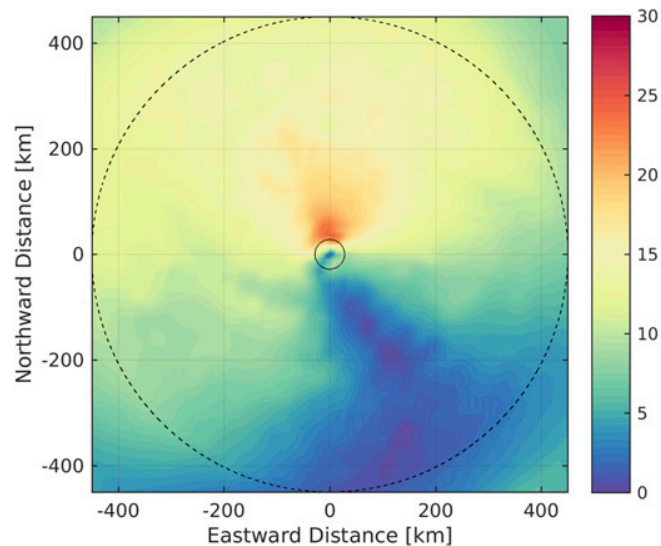

(c) $\triangle R M W:-20 \%$

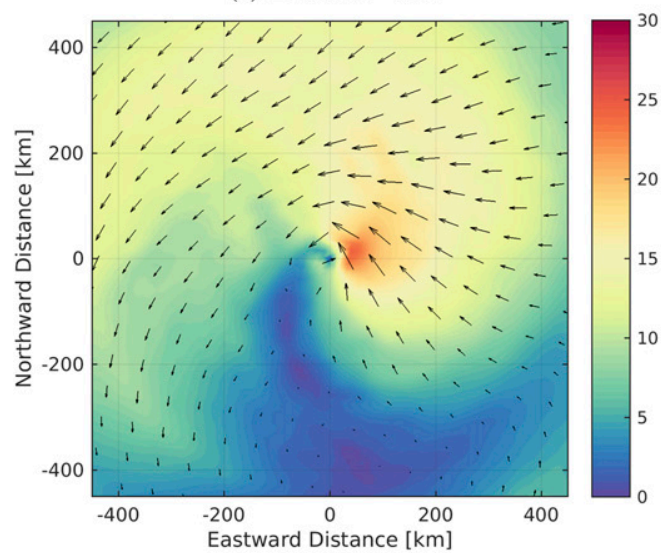

(e) $\theta:-90^{\circ}$

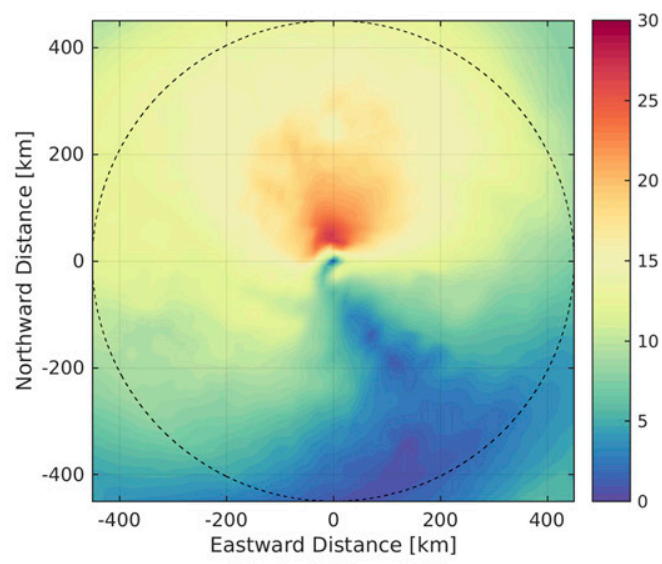

(b) $\Delta A_{0}^{\max }:+20 \%$

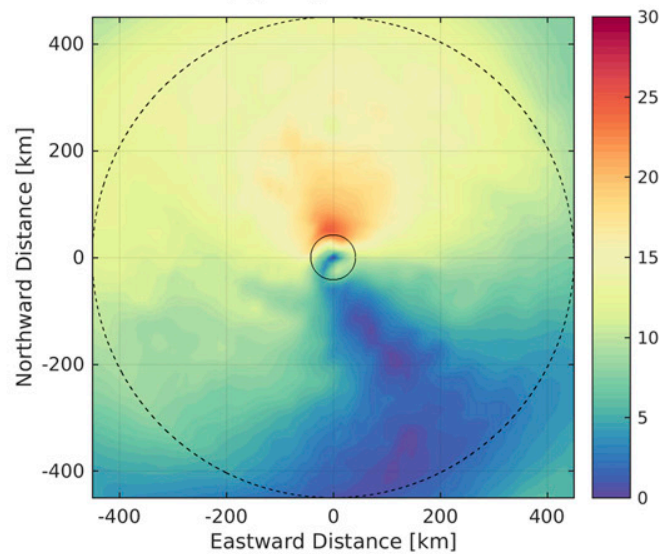

(d) $\triangle R M W:+20 \%$

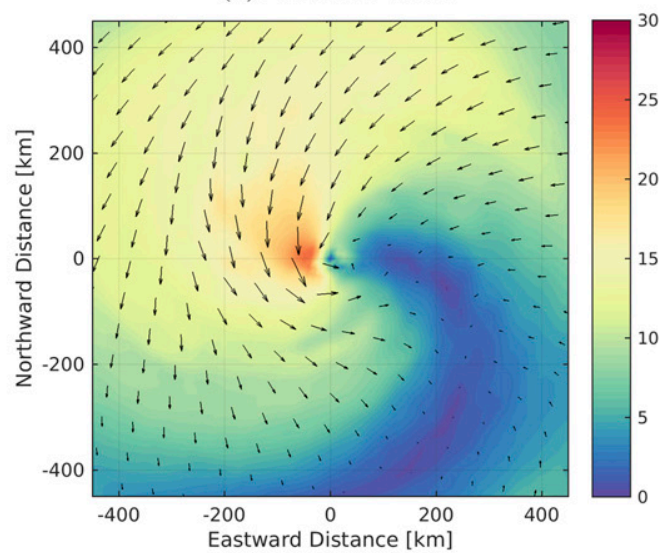

(f) $\theta:+90^{\circ}$

FIG. 2. Examples of initial fields resulting from univariate parameter perturbations. (top) Storm intensity perturbations, (middle) storm size perturbations, and (bottom) storm rotation perturbations. Refer to Fig. 1 for the unperturbed field.

modeling (OGCM) (Alexanderian et al. 2012; Thacker et al. 2012; Iskandarani et al. 2016a; Li et al. 2016; Sraj et al. 2016; Sraj et al. 2013). This subsection provides a brief outline of the PC framework used in the present study.
Let $\boldsymbol{\xi}=\left(\xi_{1}, \xi_{2}, \ldots, \xi_{4}\right)^{\mathrm{T}}$ be the four-dimensional random vector, whose components are independent random variables uniformly distributed over $[-1,1]$. These canonical random variables parameterize the uncertainty in the initial storm properties as reflected in 

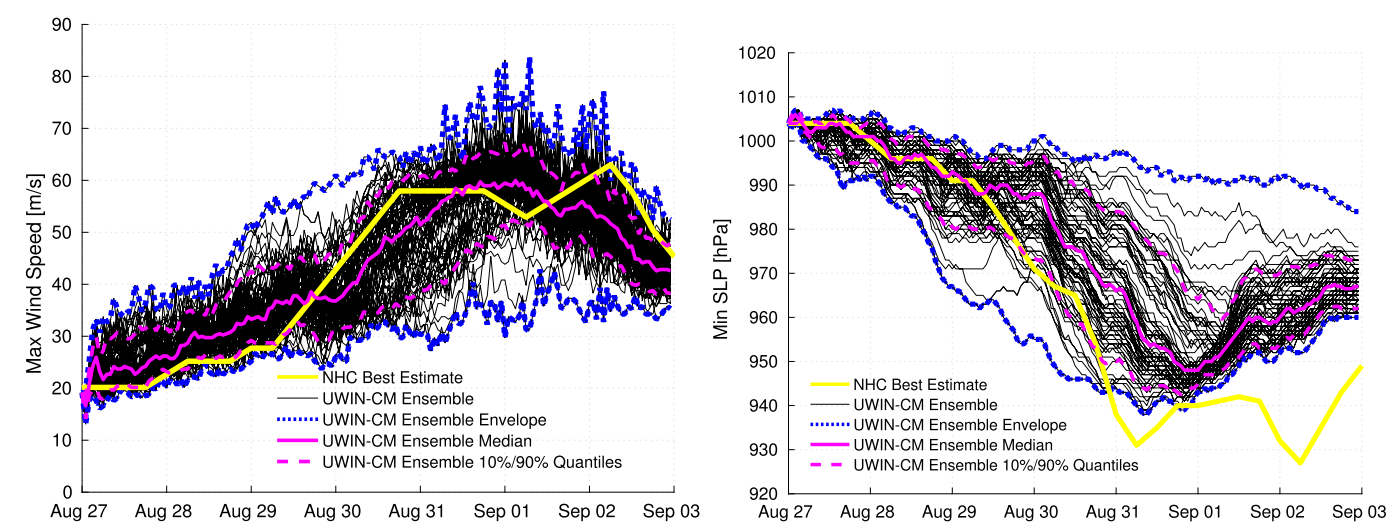

FIG. 3. UWIN-CM model realizations (black solid lines) of the time evolution of (left) MWS and (right) MSLP. Plotted are curves showing the NHC best estimates (yellow), the UWIN-CM ensemble envelope (dashed blue), the UWIN-CM median (solid magenta), and the 10\%/90\% UWIN-CM ensemble quantiles (dashed magenta).

Table 1. In addition, let $\mathscr{Q}(\boldsymbol{\xi})$ be a real-valued QoI obtained from the UWIN-CM simulation corresponding to a given random input $\boldsymbol{\xi}$. Under the assumption of finite variance (Le Maître and Knio 2010), $\mathscr{Q}(\boldsymbol{\xi})$ can be approximated by the following truncated Fourier-like expansion:

$$
\mathscr{Q}(\boldsymbol{\xi}) \approx \tilde{\mathscr{Q}}(\boldsymbol{\xi})=\sum_{\alpha=0}^{N_{p}} c_{\alpha} \Psi_{\alpha}(\boldsymbol{\xi}),
$$

where $c_{\alpha} \in \mathbb{R}$ are the expansion coefficients, $\Psi_{\alpha}: \xi \in$ $\Xi \mapsto \mathbb{R}$ are orthogonal multivariate Legendre polynomials in $\boldsymbol{\xi}$, and $\left(N_{p}+1\right)$ is the total number of polynomials retained in the expansion (i.e., the truncated basis). To determine the unknown coefficients $c_{\alpha}$, we solve an $\ell_{1}$ regularized regression problem (Peng et al. 2014; Li et al. 2016), using as data the UWIN-CM simulation ensemble $\left(\mathscr{P}_{\mathrm{LHS}}^{80}\right)$ described above. The regularized regression methodology enables us to consider a truncated basis (or library) whose size is larger than the number of available realizations. In the applications below, we use a PC library corresponding to a polynomial basis truncated at total order eight; with a four-dimensional random vector, the library contains 495 polynomials including the constant term (i.e., $\left.N_{p}+1=495\right)$.

The PC representation [Eq. (4)] readily affords calculations of various statistical moments, such as mean and variance that can be estimated, respectively, from

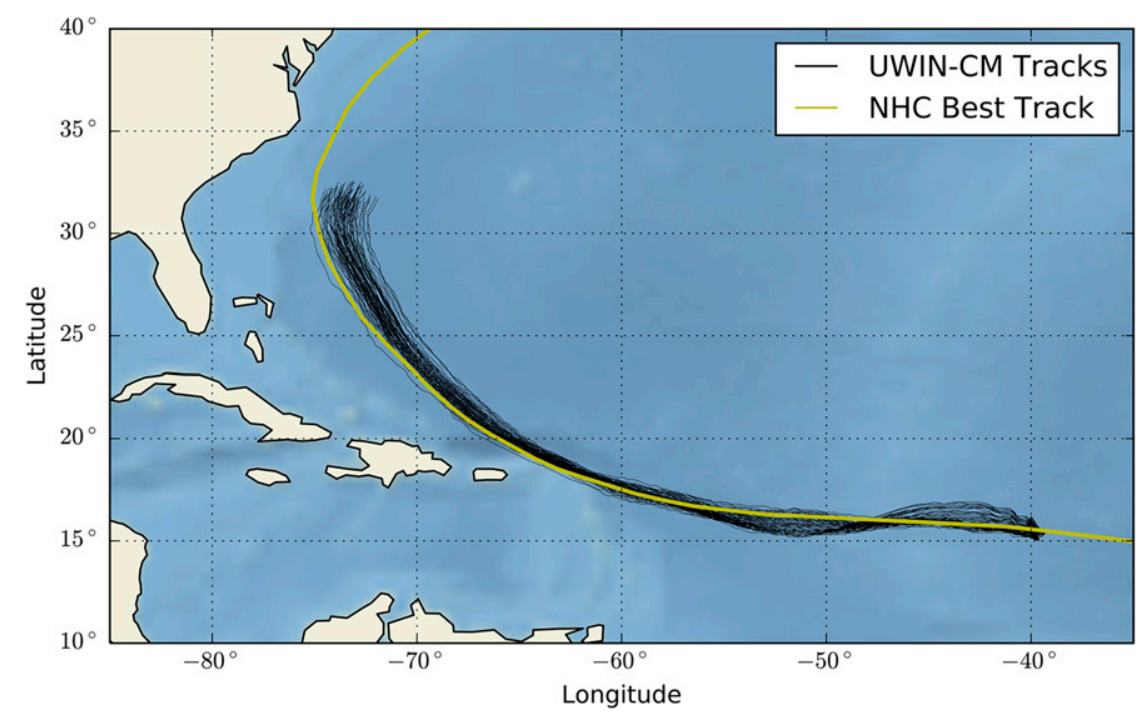

FIG. 4. UWIN-CM model realizations of storm tracks. The yellow curve represents the best track estimates by NHC. 


$$
\mathbb{E}[\tilde{\mathscr{Q}}]=\sum_{\alpha=0}^{N_{p}} c_{\alpha}\left\langle\Psi_{\alpha}, 1\right\rangle=c_{0},
$$

and

$$
\begin{aligned}
\mathbb{V}[\tilde{\mathscr{Q}}] & =\mathbb{E}\left[(\tilde{\mathscr{Q}}-\mathbb{E}[\tilde{\mathscr{Q}}])^{2}\right]=\sum_{\alpha, \beta=1}^{N_{p}} c_{\alpha} c_{\beta}\left\langle\Psi_{\alpha}, \Psi_{\beta}\right\rangle \\
& =\sum_{\alpha=1}^{N_{p}} c_{\alpha}^{2}\left\|\Psi_{\alpha}\right\|_{L_{2}}^{2}
\end{aligned}
$$

[refer to Le Maître and Knio (2010) for more details]. It also enables us to estimate the variance-based, firstorder and total-order sensitivity indices (Sobol 1993; Homma and Saltelli 1996) associated with random variable $\xi_{i}$, namely using

$$
\begin{aligned}
\mathbb{S}_{i}=\frac{\sum_{\alpha \in \mathscr{S}_{i}} c_{\alpha}^{2}\left\|\Psi_{\alpha}\right\|_{L_{2}}^{2}}{\sum_{\alpha=1}^{N_{p}} c_{\alpha}^{2}\left\|\Psi_{\alpha}\right\|_{L_{2}}^{2}}, \\
\mathbb{T}_{i}=\frac{\sum_{\alpha \in \mathscr{T}} c_{\alpha}^{2}\left\|\Psi_{\alpha}\right\|_{L_{2}}^{2}}{\sum_{\alpha=1}^{N_{p}} c_{\alpha}^{2}\left\|\Psi_{\alpha}\right\|_{L_{2}}^{2}},
\end{aligned}
$$

where $\mathscr{S}_{i}\left(\mathscr{T}_{i}\right)$ is the set of polynomial indices such that $\Psi_{\alpha}(\alpha>0)$ has degree 0 in all random variables other than $\xi_{i}$ (which has degree $>0$ in $\xi_{i}$ ). Recall that the firstorder sensitivity index associated with $\xi_{i}$ only accounts for the variance arising from monomials of $\xi_{i}$, whereas the total order sensitivity index in addition includes variance contributions arising from cross terms (or interactions) between $\xi_{i}$ and other random variables.

The calculations of the PC coefficients for time- and space-dependent QoI require the repeated solution of the $\ell_{1}$ regularized regression problem for each spacetime location. This represents a significant computational burden and the difficulty is compounded when the QoI exhibits high-frequency oscillations such as those found in the max wind speed and the MSLP (see Fig. 3). These difficulties can be overcome by adopting a dimensionality reduction approach that combines empirical orthogonal function (EOF) decomposition and the PC methodology, as done in Li et al. (2016). The EOF-based PC surrogate model for a time-dependent QoI can be expressed as

$$
\begin{aligned}
Q(t, \boldsymbol{\xi}) & =\bar{Q}(t)+\sum_{m=1}^{M} \sqrt{\lambda_{m}} q^{m}(t) \phi^{m}(\boldsymbol{\xi}) \\
& =\bar{Q}(t)+\sum_{m=1}^{M} \sqrt{\lambda_{m}} q^{m}(t) \sum_{\alpha=0}^{N_{p}} c_{\alpha}^{m} \Psi_{\alpha}(\boldsymbol{\xi}),
\end{aligned}
$$

where $M$ is the number of modes retained in the EOF expansion, and $\bar{Q}(t)=1 / N_{r l z} \sum_{k=1}^{N_{r l z}} \mathscr{Q}\left(t, \boldsymbol{\xi}_{k}\right)$ is the simulation ensemble mean. The term $\left[\lambda_{m}, q^{m}(t)\right]$ denotes the $m$ th eigenvalue-eigenvector pair in the EOF decomposition of $\mathcal{Q}(t, \boldsymbol{\xi})$, and $\phi^{m}(\boldsymbol{\xi})$ is the corresponding random coefficient. Following Li et al. (2016), $\phi^{m}$ is approximated by the PC expansion $\sum_{\alpha=0}^{N_{p}} c_{\alpha}^{m} \Psi_{\alpha}(\xi)$.

Letting $C_{\alpha}(t)=\sum_{m=1}^{M} \sqrt{\lambda_{m}} q^{m}(t) c_{\alpha}^{m}$, the EOF-PC representation of $\mathscr{Q}(t, \boldsymbol{\xi})$ can be expressed as

$$
\mathscr{Q}(t, \boldsymbol{\xi})=\bar{Q}(t)+\sum_{\alpha=0}^{N_{p}} C_{\alpha}(t) \Psi_{\alpha}(\boldsymbol{\xi}),
$$

which has the same structure as Eq. (4) (except for the additional term corresponding to the ensemble mean). As a result, the formulas for the statistical moments and sensitivity indices can be directly applied to Eq. (9).

Compared with a direct PC approximation of a QoI, the EOF-PC decomposition involves a significantly reduced dimensionality. Specifically, the latter involves $M$ time-dependent eigenvectors whereas the former involves 168 hourly outputs. More importantly, by projecting the response onto the $M$-dimensional space spanned by the EOFs, a regularization effect is implicitly achieved, as manifested by suppression of high-frequency oscillations and a loss in variability.

\section{b. Uncertainties in EOF decomposition}

Before moving on to the $\mathrm{PC}$ analysis, we first determine the impact of the EOF truncation parameter $M$ in Eq. (8) by examining the cumulative variability associated with the first few leading EOF modes of both MWS and MSLP. Figure 5 shows these two curves up to mode five.

It is seen that for the MSLP response, because of its relatively smooth time evolution, its cumulative variance curve converges rapidly, with more than $95 \%$ of variance captured in the first five modes. On the other hand, the MWS response, which may be affected by convective bursts, exhibits a great amount of high-frequency variability so that the cumulative variance convergence rate is much slower and the variance curve starts to flatten at mode 5 with around $78 \%$ variance explained. Despite the relatively low level of explained variance, our numerical experiments suggest that retaining five EOF modes for MWS response is still a reasonable choice (as higher modes fail to bring noticeable improvement in our EOF expansion). As a result, we retain five modes for both the wind and SLP responses.

Traditional EOF analysis ignores underlying uncertainties in eigendecomposition when dealing with random sample data. However, the high-frequency variability in the temporal evolution of MWS (see Fig. 3) and the limited number of model realizations 


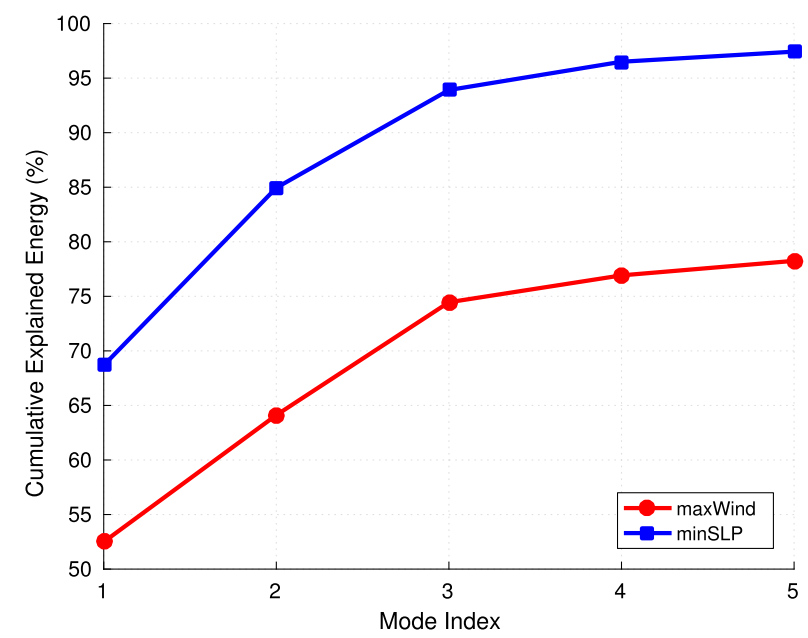

FIG. 5. Cumulative energy captured by EOF decomposition up to mode five.

necessitate careful examination of uncertainties in EOF decompositions of both MWS and MSLP responses. To this end, the Bayesian Karhunen-Loève (KL) procedure proposed by Chowdhary and Najm (2016) is utilized. The

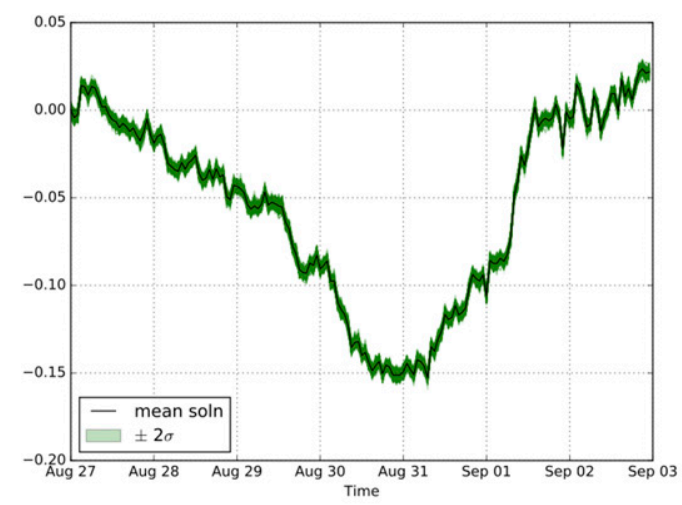

(a) maxWind, Mode-1

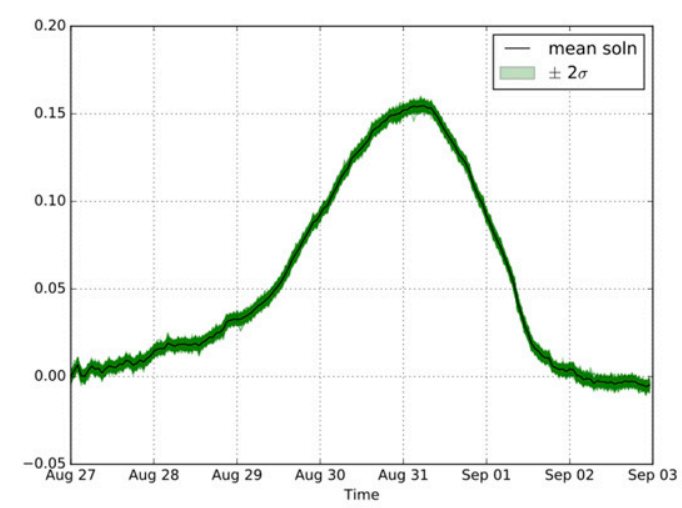

(c) minSLP, Mode-1
Bayesian KL approach essentially generalizes the classical EOF decomposition framework, namely by accommodating uncertainties in the EOF themselves. This is achieved by applying an extended Bayesian inference exercise, in which a statistical sampling is applied to simultaneously infer uncertain modes and uncertain mode amplitudes from the ensemble simulation results. The resulting posterior thus enables us to quantify the uncertainty in the orthogonal modes, and consequently assess the robustness of the decomposition. Figure 6 shows the first two eigenmodes of both MWS and MSLP, with their corresponding $\pm 2 \sigma$ probability bounds. It is seen from the tight $\pm 2 \sigma$ intervals that the uncertainty in eigendecomposition is relatively small, which lends confidence in our EOF formalism despite the limited ensemble size.

In addition, the Bayesian KL procedure of Chowdhary and Najm (2016) allows resampling of the underlying QoIs by design, leading to the ability of examining probability distribution of QoI responses. Figure 7 compares probability boundaries from Bayesian KL resampling results with UWIN-CM ensemble $\left(\mathscr{P}_{\text {LHS }}^{80}\right)$ outputs. The probability boundaries are defined by

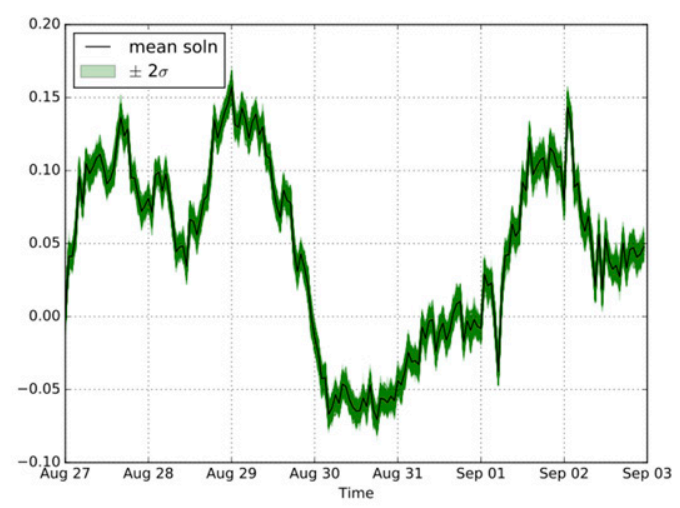

(b) maxWind, Mode-2

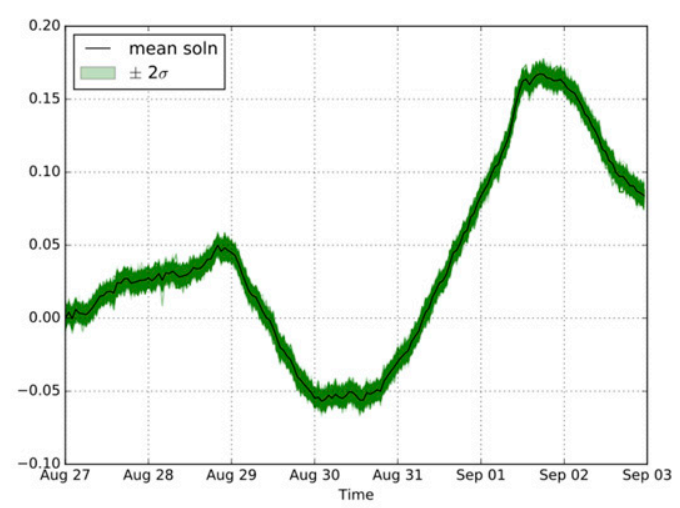

(d) $\operatorname{minSLP}$, Mode-2

FIG. 6. First two eigenmodes of each QoI (as indicated) in EOF decomposition and the corresponding $\pm 2 \sigma$ bounds. 


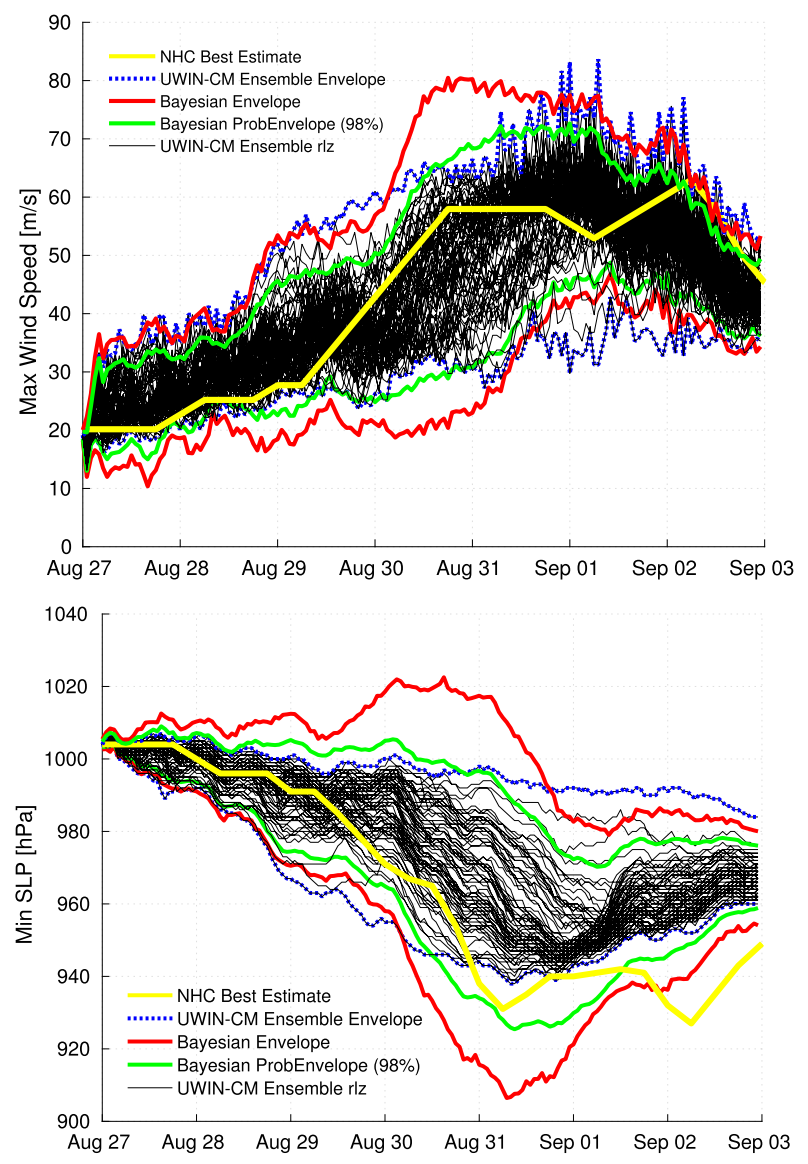

FIG. 7. Probability boundaries of Bayesian KL resampling of both (top) MWS and (bottom) MSLP, along with UWIN-CM ensemble results and the best NHC estimates (yellow curves). Red curves are absolute bounds of Bayesian KL resampling, and green curves indicate the $98 \%$ confidence interval from Bayesian KL resampling. The blue dotted lines denote the UWIN-CM realization bounds.

$$
\left\{\begin{array}{l}
\mathscr{Q}^{+}(t, p)=\mathscr{Q}(t) \text { s.t. } \operatorname{Prob}\left[\mathscr{Q}_{\mathrm{BKL}}(t)>\mathscr{Q}(t)\right]=p \\
\mathscr{Q}^{-}(t, p)=\mathscr{Q}(t) \text { s.t. } \operatorname{Prob}\left[\mathscr{Q}_{\mathrm{BKL}}(t)<\mathscr{Q}(t)\right]=p
\end{array}\right.
$$

where $\mathcal{Q}_{\mathrm{BKL}} \mathrm{S}$ are Bayesian KL samples. When $p=0$, the above bounds correspond to the "absolute" bounds in Fig. 7, and $p=0.01$ leads to the $98 \%$ probability boundaries. Note that the above bounds are defined in a pointwise manner along the temporal axis, meaning the resulting probability boundary curves are unlikely to originate from individual realizations. Nevertheless, these probability boundaries provide insightful statistics on temporal responses of MWS and MSLP.

It is observed from Fig. 7 that the absolute boundaries enclose almost all UWIN-CM realizations, with only one or two realization curves escaping from the absolute envelope during 29-30 August and 1-3 September. Note that the absolute boundaries exhibit significant over and/or under shoot between 30 August and 1 September. Because these absolute boundaries are of extremely low probability (lying within the tails of the underlying probability distributions), a better approach would be to cut off the low probability tails and examine the $98 \%$ percentile support boundaries, as shown by the green curves in Fig. 7. As can be seen, these $98 \%$ percentile boundaries fit tightly to the UWIN-CM realizations, and reduce the "unrealistic" peak/minimum in absolute boundaries at the expense of allowing a few more realizations to escape from the $98 \%$ boundaries. The probability of UWIN-CM model response (either the MWS or MSLP) falling between the $98 \%$ confidence intervals from Bayesian KL resampling in Fig. 7 is about $98 \%$. Additional discussion on model prediction statistics follows below.

\section{c. PC surrogate results}

As the Bayesian KL analysis above shows that the EOF modes of time evolutions of both MWS and MSLP are suitably estimated, we move on to the analysis of PC surrogate models [in the form of Eq. (9)] of the two QoIs, and compare PC predicted results against UWIN$\mathrm{CM}$ realizations. Figure 8 shows $\mathrm{PC}$ predictions at 0000 UTC 1 September, which are compared with UWIN-CM realization histograms. The yellow vertical lines indicate the best estimate of the corresponding QoI at the given time by NHC. It is clear that our PC predictions closely resemble the UWIN-CM realizations in the distribution sense, including the overall distribution shapes, and peak locations. However, PC-predicted distributions do miss a few outliers; for example, the lower bound (about $30 \mathrm{~m} \mathrm{~s}^{-1}$ ) and upper bound (about $80 \mathrm{~m} \mathrm{~s}^{-1}$ ) realization instances in the MWS plot, and the upper bound (about $980-990 \mathrm{hPa}$ ) instances in the MSLP plot.

In addition, we generate an independent 11-member UWIN-CM simulation ensemble (denoted as $\mathscr{P}_{\text {valid }}^{11}$ hereafter) with univariate perturbation along the RMW parameter axis (while setting other random parameters to zero), and further validate our PC prediction capability on this validation set. Figure 9 shows the comparison of MSLP response curves between UWIN-CM realizations and PC predictions on $\mathscr{P}_{\text {valid }}^{11}$. Observe that UWIN$\mathrm{CM}$ realizations in the two bottom-left panels correspond to two early intensified storms. Though the PC surrogate model evidently exhibited discrepancies in reproducing outliers, it successfully predicted the evolution of the MSLP in most cases. In particular, the minimum values are well predicted [within $10(\mathrm{hPa})$ error]. The comparison results for MWS responses are similar, except for the fact that the curves exhibit more oscillations as one would expect. These are omitted for brevity. 

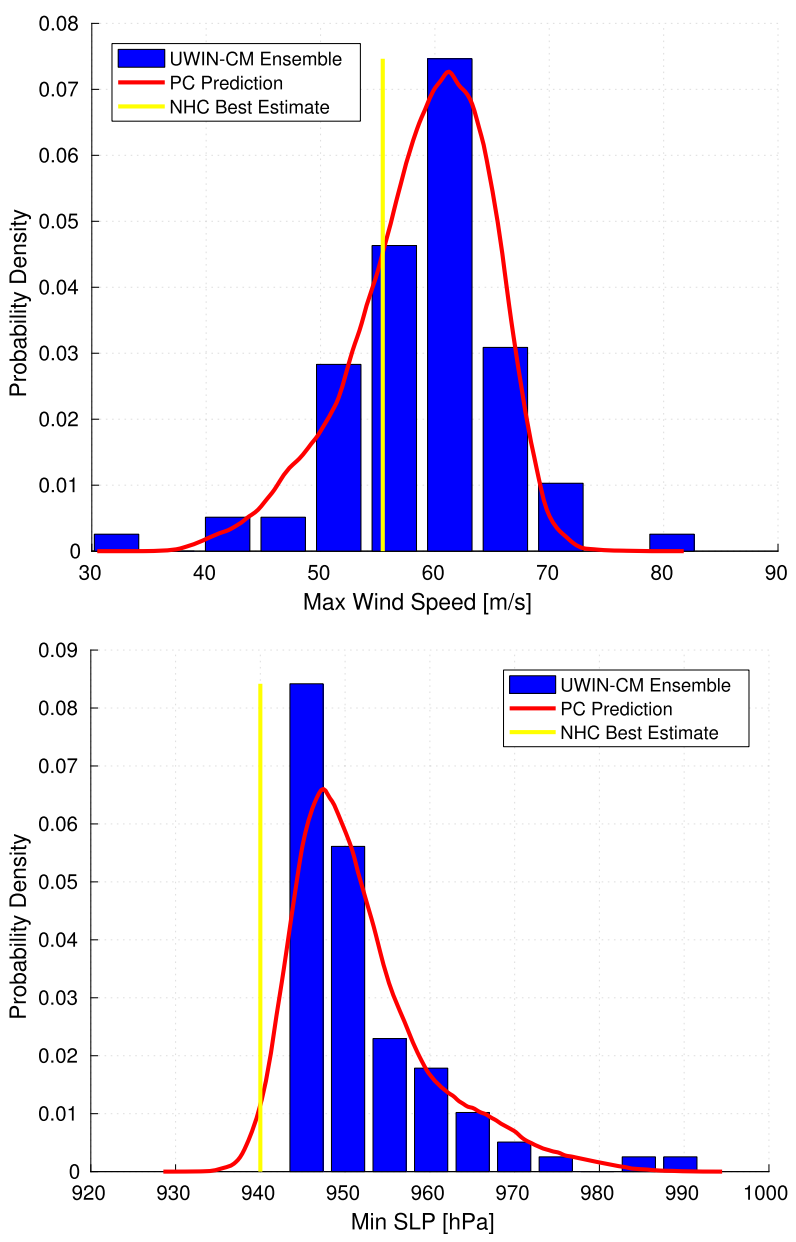

FIG. 8. Probability distribution of (top) MWS and (bottom) MSLP at 0000 UTC 1 Sep. PC prediction curves are obtained via KDE analysis on PC evaluations over an LHS set of $10^{6}$ random samples, while the histograms are obtained from the 80 UWIN-CM realizations.

It is worth noting that the stochastic perturbations introduced via the parameterization in section $2 \mathrm{~b}$ are not the only random source in our UWIN-CM simulations. Compiler configurations, parallelization, and machine precision error (these are referred to as intrinsic errors hereafter) also have a "stochastic" effect on model responses. In other words, the UWIN-CM outputs are not strictly deterministic and subject to stochastic behavior stemming from those intrinsic errors. [Combined with complex sensitivity of the system to changes in initial conditions, this generally leads us to expected irreducible residuals in surrogate representations of QoIs (Sraj et al. 2016).] Additional numerical experiments suggest that though they cannot be avoided, intrinsic errors usually lead to relatively small variability in model responses. This is also partially supported by the relatively tight SLP band in the bottom-right panel of Fig. 9. For the time being, we put aside the concern of potential impact of intrinsic model errors, which lead to the lack of repeatability of UWIN-CM predictions, and move forward with the PC statistical analysis.

Figure 10 shows probability envelopes given by PC surrogates, for both MWS (top panel) and MSLP (bottom panel). Close agreement between PC predicted probability boundaries and those from Bayesian KL analysis in Fig. 7 is observed (especially the 98 percentile boundaries). This suggests that our PC surrogate models are able to reproduce the variability of the UWIN-CM ensemble. This is further supported by the time evolution of QoI response variabilities (in terms of standard deviation) as shown in Fig. 11. Both PC surrogate models and Bayesian KL resampling closely reproduce the standard deviation curves reported by empirical calculations of UWIN-CM realizations on $\mathscr{P}_{\text {LHS }}^{80}$. In the MWS plot (the left panel in Fig. 11), the Bayesian KL resampling variability is close to the PC variability as EOF truncation for MWS dominates in the model error of both Bayesian KL and PC analysis. On the other hand, the EOF truncation error is much smaller in the analysis of MSLP, leading Bayesian KL resampling to better reproduce the empirical standard deviation of UWIN-CM realizations. Here the PC surrogate model suffers larger error compared with the Bayesian KL resampling (see in the right panel of Fig. 11), because of additional regularization in solving the regression problem for the PC expansion coefficients (Peng et al. 2014; Li et al. 2016).

In addition, peak model response variabilities are found around 31 August for both the MWS and MSLP (Fig. 11), which coincides with the time of fully intensified storm state as can be seen from Figs. 7 and 10. As pointed out by Judt and Chen (2016), some of the UWIN-CM realizations show rapid intensification of the underlying storm, while others do not intensify at all. The predictability analysis of Judt and Chen (2016) is extended in the next section.

\section{d. Rapid intensification prediction}

The definition of RI of TCs can be found in Kaplan and DeMaria (2003), which identifies RI storms by an at least $15 \mathrm{~m} \mathrm{~s}^{-1}$ increase in the MWS within a 24-h period. We follow the same definition to classify both our UWIN-CM realizations (on $\mathscr{P}_{\mathrm{LHS}}^{80}$ ), as well as sampling results from both Bayesian KL and PC analyses.

For clarity, we express the RI classification criterion as follows:

$$
\mathrm{RI}=\left\{\begin{array}{ll}
1 & \text { if } \exists t, \text { s.t. } w_{\text {max }}(t+24)-w_{\max }(t)>\delta W \\
0 & \text { otherwise }
\end{array},\right.
$$

where $w_{\max }(t)$ denotes the MWS at time $t$ (in hours), and $\delta W$ is the RI threshold. Table 2 shows the proportion of 

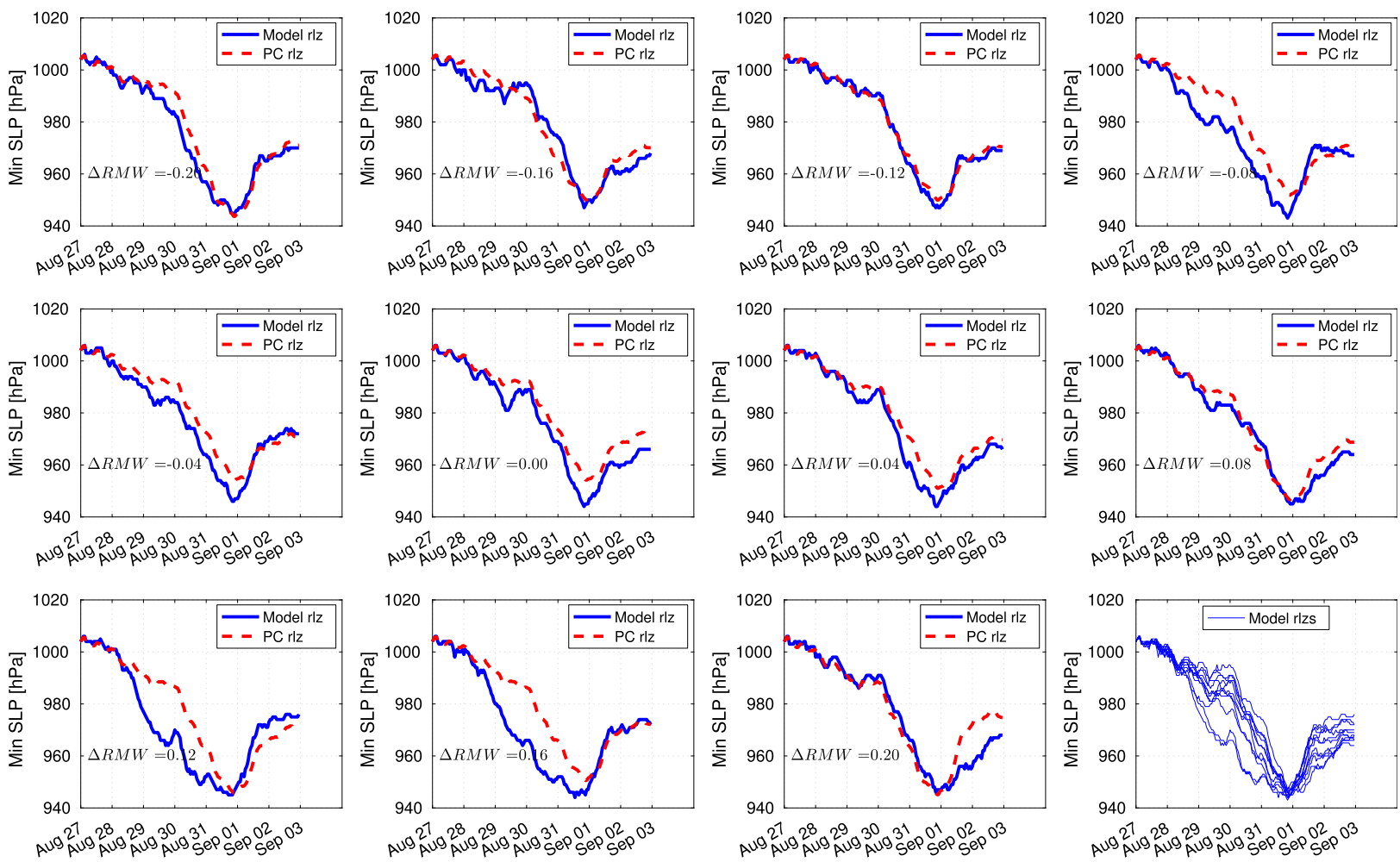

FIG. 9. Comparing PC predictions of MSLP with out-of-sample UWIN-CM model realizations ( $\left.\mathscr{P}_{\mathrm{LHS}}^{11}\right)$. The out-of-sample perturbations are along the RMW axis, other random variables are unperturbed.

RI storms in different realization sets, under the same threshold $\delta W=15 \mathrm{~m} \mathrm{~s}^{-1}$. The classification on UWIN$\mathrm{CM}$ outputs suggests that it is almost certain for the storm to undergo a rapid intensification, regardless of the initial perturbation applied. This RI rate is even higher than that reported in Judt and Chen (2016). On the other hand, UWIN-CM outputs lead to significantly higher ratios than those from Bayesian KL or EOF-PC reconstructed MWS responses. This is the result of the combined effect of mode truncation in both the Bayesian KL and EOF-PC approaches, as well as the regularization used in the regression applied to determine the stochastic mode amplitudes. As discussed by $\mathrm{Li}$ et al. (2016), both truncation (which involves projection on a finitedimensional subspace) and regularization (which limits the order of the basis functions) introduce a considerable amount of smoothing. In the present case, the smoothing effect of mode truncation (see energy estimates in Fig. 5) dominates over that of regularized regression errors (not shown). Irrespective of their relative magnitudes, a consequence of these smoothing mechanisms is that the reconstructed MWS is more likely to be classified as non-RI under the same threshold. Note that the impact of the smoothing mechanisms may be systematically reduced by retaining a larger number of modes in the expansion, and ensuring that a commensurate size ensemble is available to support building higherdimensional representations ( $\mathrm{Li}$ et al. 2016). Because of the expense of generating additional samples, however, it was not possible to conduct such refinement study in the present context.

To better understand the stochastic dependence of RI on random storm perturbations, we examine the probability distribution of observing a rapidly intensifying storm in the parameter space $\boldsymbol{\Xi}$ [i.e., $p(\mathrm{RI}=1 \mid \boldsymbol{\xi})]$. This conditional probability can be expressed by the following Bayes's formula:

$$
p(\mathrm{RI}=1 \mid \boldsymbol{\xi}) \propto p(\boldsymbol{\xi} \mid \mathrm{RI}=1) \times p(\mathrm{RI}=1),
$$

where $p(\mathrm{RI}=1)$ is the prior probability distribution of a storm undergoing RI. As discussed above, this probability is highly dependent on the RI threshold $\delta W$. Nevertheless, if the RI threshold is fixed, then $p(\mathrm{RI}=1)$ is simply a constant, and the above Bayes's formula reduces to

$p(\mathrm{RI}=1 \mid \boldsymbol{\xi}) \propto p(\boldsymbol{\xi} \mid \mathrm{RI}=1) \quad$ with fixed RI threshold.

To calculate the conditional probability distribution $p(\boldsymbol{\xi} \mid \mathrm{RI}=1)$ (i.e., the probability distribution of initial 

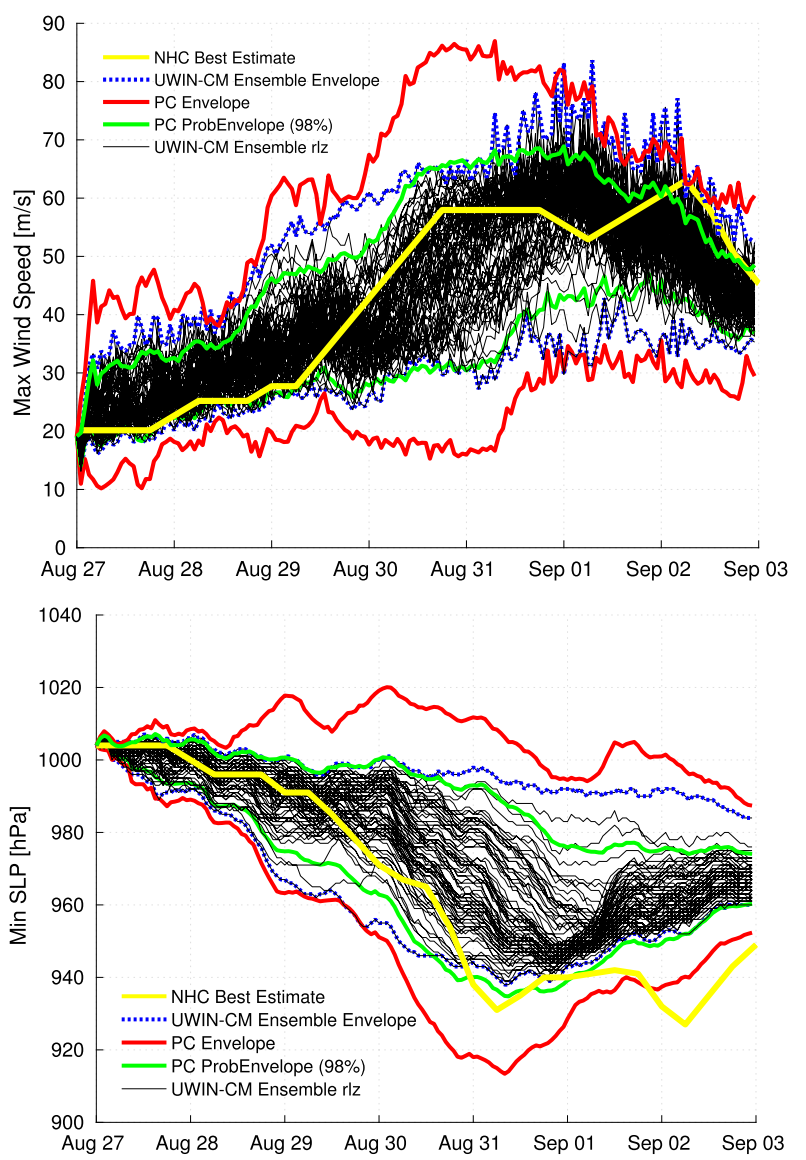

FIG. 10. Probability boundaries given by PC surrogate models for both (top) MWS and (bottom) MSLP, along with UWIN-CM ensemble results and the best NHC estimates (yellow curves). Red curves are absolute PC prediction bounds, and green curves indicate the $98 \%$ confidence interval from PC sampling. The blue dotted lines denote the UWIN-CM realization bounds.

random parameter $\boldsymbol{\xi} \in \Xi$, provided a storm undergoes $\mathrm{RI})$, we use the same EOF-PC resampling set $\left(10^{6}\right.$ samples drawn randomly) as used for obtaining the probability boundaries in Fig. 10, and filter out all RI realizations and their corresponding random inputs $(\xi \mathrm{s})$ with respect to different thresholds $(\delta W \in\{10$, $11, \ldots, 15\} \mathrm{m} \mathrm{s}^{-1}$ ). Kernel density estimation (KDE) analysis is then applied to the filtered random inputs to generate probability density distributions $p(\xi \mid \mathrm{RI}=1)$. Note the distributions (small-scale details in particular) obtained from KDE analysis largely depend on the choice of kernel size. Because of the lack of ground truth reference, we only focus on large-scale distribution patterns that are more stable with respect to kernel size. Figures 12-14 show marginal distributions in different 2D parameter spaces (along with 1D marginal probability distributions of corresponding random variables), with respect to different thresholds as indicated. For clarity, the marginal distribution in $\xi_{1}-\xi_{4}$ subspace reads as follows:

$$
\begin{aligned}
& p\left(\xi_{1}, \xi_{4} \mid \mathrm{RI}=1\right) \\
& \quad=\frac{1}{4} \int_{\xi_{2} \in[-1,1]} \int_{\xi_{3} \in[-1,1]} p\left(\xi_{1}, \xi_{2}, \xi_{3}, \xi_{4} \mid \mathrm{RI}=1\right) d \xi_{2} d \xi_{3} .
\end{aligned}
$$

Figure 12 shows marginal distributions of $p(\boldsymbol{\xi} \mid \mathrm{RI}=1)$ in the $\xi_{1}-\xi_{4}$ subspace, which corresponds to average storm strength $\left(\xi_{1}\right)$ and rotation $\left(\xi_{4}\right)$ perturbations (see Table 1), respectively. It is seen that despite the different values of RI thresholds and different color scales, the overall marginal distributions (spatial patterns of high/ low probability regions) look very similar, suggesting this distribution analysis is stable with respect to the threshold. Furthermore, it is evident that the probability of RI increases with positive perturbations in the storm strength and rotation. Thus, we conclude that RI storms are more likely the results of stronger initial intensification with counterclockwise rotation perturbations. In addition, we observe similar variability of the marginal probability density along both the $\xi_{1}$ and $\xi_{4}$ axes, suggesting their comparable associated sensitivities.
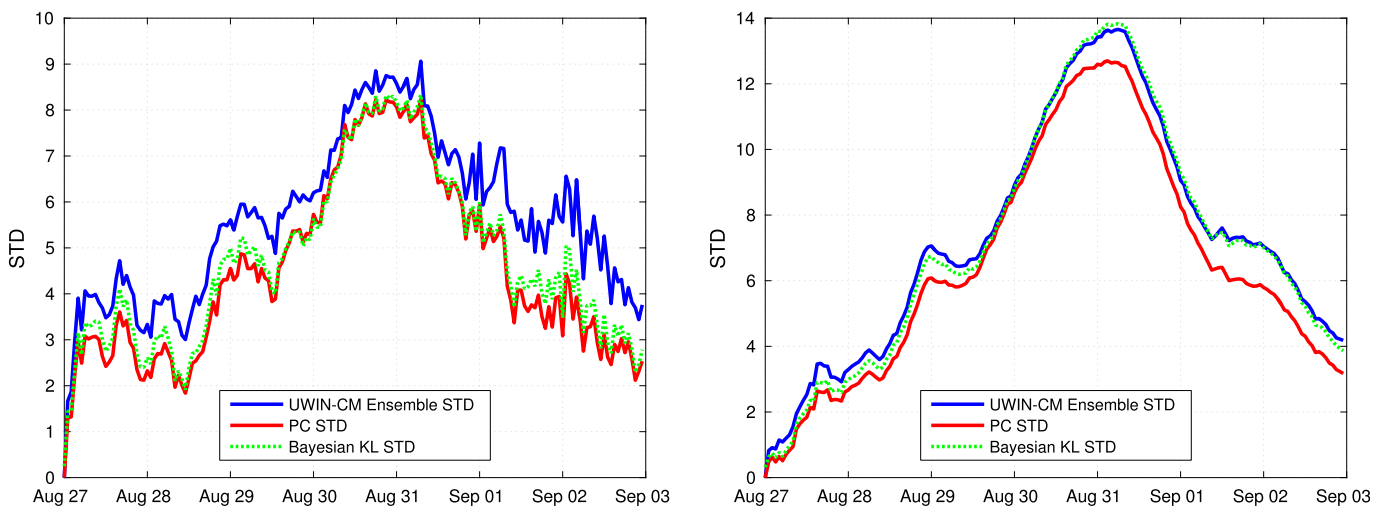

FIG. 11. Time evolution of model response uncertainties: (left) MWS and (right) MSLP. 
TABLE 2. Proportion of RI realizations in different realization sets, under threshold of $\delta W=15 \mathrm{~m} \mathrm{~s}^{-1}$ over a 24 -h period; $\mathscr{P}{ }_{\mathrm{LHS}}^{80}$ denotes the original 80 -member ensemble, while $\mathscr{P}_{\text {valid }}^{11}$ denotes the additional validation set in Fig. 9 . Resampling sets are those sample sets used to generate the probability boundaries in Figs. 7 and 10.

\begin{tabular}{lccc}
\hline \hline Sample set & UWIN-CM $\mathscr{P}_{\text {LHS }}^{80}$ & UWIN-CM $\mathscr{P}_{\text {valid }}^{11}$ & Baysian KL resampling \\
\hline Sample set & $97.5 \%$ & $100 \%$ & $54.8 \%$ \\
\hline & PC evaluation on $\mathscr{P}_{\text {LHS }}^{80}$ & PC evaluation on $\mathscr{P}_{\text {valid }}^{11}$ & PC resampling \\
\hline
\end{tabular}

In Fig. 13, similar distributions are once again observed across different thresholds, and RI storms clearly favor positive perturbations of storm rotation $\left(\xi_{4}\right)$ over the RMW $\left(\xi_{3}\right)$. In fact, there seem to be little difference between positive and negative RMW perturbations, despite a bimodal structure along $\xi_{3}$ in the upper half of the domain (positive storm rotation perturbation region). Overall, larger variation is observed along $\xi_{4}$ axis compared to that along $\xi_{3}$, suggesting higher sensitivity associated with $\xi_{4}$.

Finally, Fig. 14 shows marginal distribution in the $\xi_{1}-\xi_{3}$ space, which supports our findings above (i.e., the distributions remain consistent across different thresholds), and initial storm intensification tends to increase the probability of RI occurrence. In addition, the larger variability across $\xi_{1}$ suggests its higher associated sensitivity and larger impact on RI, compared with storm size (RMW) perturbations.

To summarize, Figs. 12-14 clearly show that the storm evolution is primarily influenced by initial storm intensity (mean wind strength component) and rotation perturbations. These two perturbations seem to exhibit comparable impact; on the other hand, RMW perturbation seems to only have a secondary impact on storm intensification. In addition, initially intensified storms (increasing $\xi_{1}$ ) with counterclockwise rotation perturbations (increasing $\xi_{4}$ ) are more likely to undergo RI.
Note that for brevity, we omit the discussion of RI probability distributions associated with $\xi_{2}$ (asymmetry part of wind strength) because they have similar behavior to $\xi_{1}$, but smaller impact on the storm intensification.

The sensitivity-related findings are also supported by the global sensitivity analysis shown in Fig. 15, which clearly indicates the dominant impact of azimuthally averaged maximum wind and asymmetry azimuth perturbations, and minor or secondary influence of the other two parameters. Specifically, the sensitivity is dominated by the asymmetry azimuth perturbations during the first day of the simulation (27-28 August). On the second and third day (28-30 August), the sensitivity to maximum azimuthally averaged wind speed takes over. These two periods are coincidental with those of low and high values of environmental wind shear, respectively. The sensitivity of MWS to storm asymmetry azimuth was high in the weakly sheared environment (up to $4 \mathrm{~m} \mathrm{~s}^{-1}$ ). However, on 28 August, the environmental shear quickly ramped up to $10 \mathrm{~m} \mathrm{~s}^{-1}$ [see Fig. 2a in Rogers et al. (2015)], which is about two standard deviations higher than the climatologically expected shear (Kaplan et al. 2010). This suggests that the environmental shear dominates the evolution of the storm intensity at this time, and is not sensitive to the asymmetry azimuth of a weak initial storm. The relative
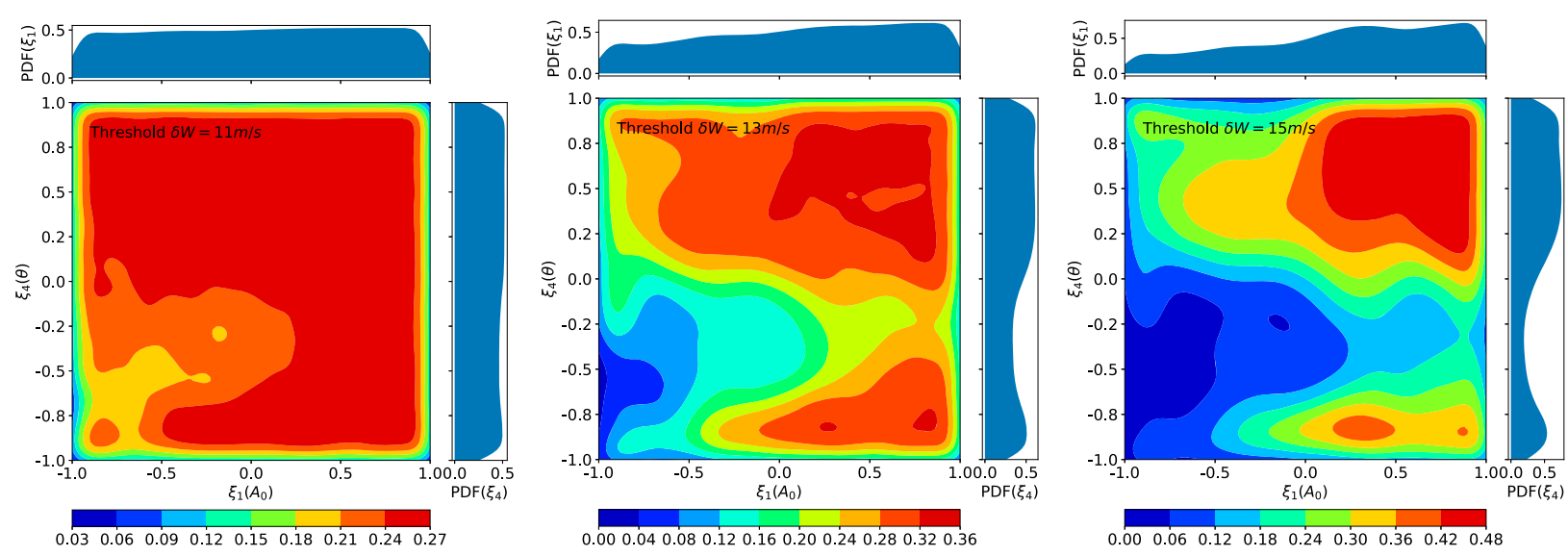

FIG. 12. Marginal and conditional probability distributions $p\left(\xi_{1}, \xi_{4} \mid \mathrm{RI}=1\right)$ of RI storm in the parameter space $\xi_{1}-\xi_{4}$ (associated with initial storm's intensity $A_{0}$ and rotation $\theta$, respectively). The threshold is varied from 11 to $15 \mathrm{~m} \mathrm{~s}^{-1}$, as indicated. 

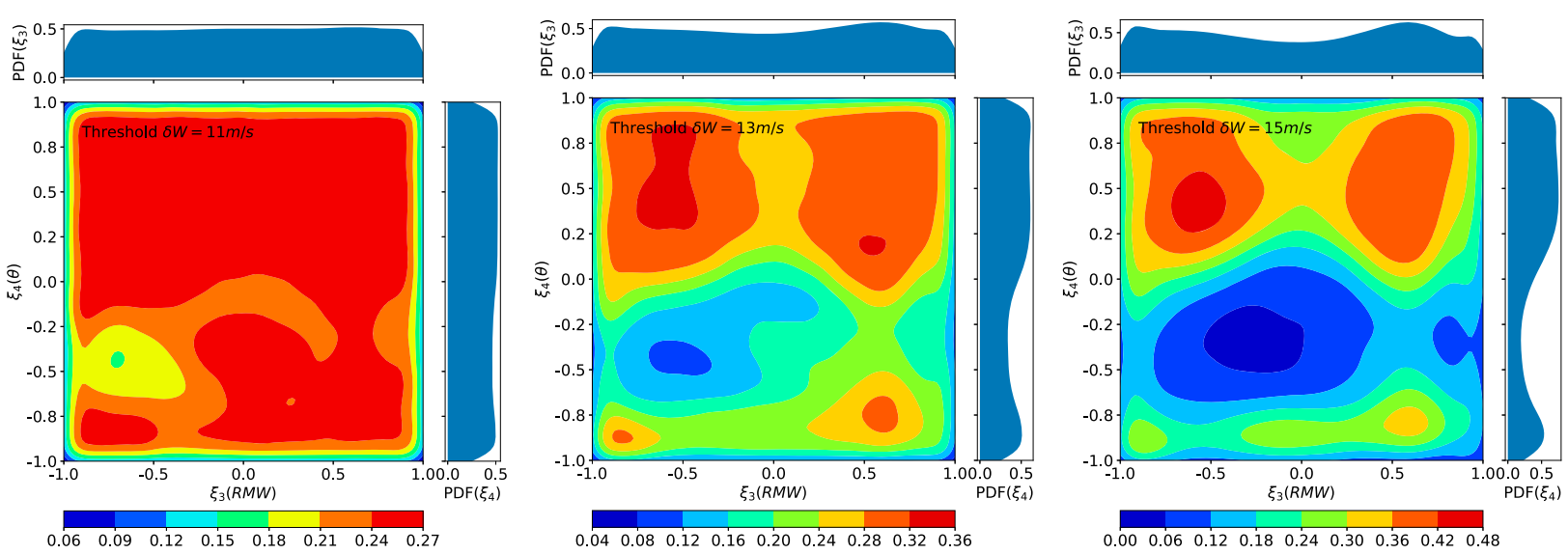

FIG. 13. Marginal and conditional probability distributions $p\left(\xi_{3}, \xi_{4} \mid \mathrm{RI}=1\right.$ ) of RI storm in the parameter space $\xi_{3}-\xi_{4}$ (associated with initial storm's RMW and rotation $\theta$, respectively). The threshold is varied from 11 to $15 \mathrm{~m} \mathrm{~s}^{-1}$, as indicated.

sensitivity to different vortex parameters thus is highly variable in time and possibly strongly depends on the storm environment. This warrants further investigation of sensitivity in other TC cases.

\section{Semiparametric simulations}

In this section, we combine the initial condition perturbations discussed earlier with SKEBS forcing used in Judt and Chen (2016) to examine the relative impact of the two perturbation mechanisms on storm evolution. In light of the sensitivity analysis in the previous section, we perturb only the two dominant parameters (initial average intensity parameter $\xi_{1}$ and asymmetry azimuth $\xi_{4}$ ), while fixing the other two parameters at zero. A 10-member LHS design in the two-dimensional space $\left(\xi_{1}-\xi_{4}\right)$ is used to sample the storm's mean wind speed intensity and rotation, and for each realization of the
10 initial storm samples, we run three SKEBS forced UWIN-CM simulations. As a result, a total of 30 UWIN-CM realizations are generated (Figs. 16 and 17). Note that the bottom-right panel of Fig. 17 depicts the individual realizations of the 10-member LHS design, and that for each of the corresponding initially perturbed fields three SKEBS simulations are performed. Each of the stochastically forced simulations uses a different value of the random seed.

The response in MWS and MSLP (Fig. 16) closely matches that of the initial condition realization ensemble (Fig. 3), except for a few nonintensified outliers. Further comparison between realization ensembles in this study and those reported in Judt and Chen (2016) suggests that despite the different mechanisms in the parametric design of storm perturbation and the SKEBS random forcing perturbation, they both lead to similar MWS and MSLP response variability. On the other
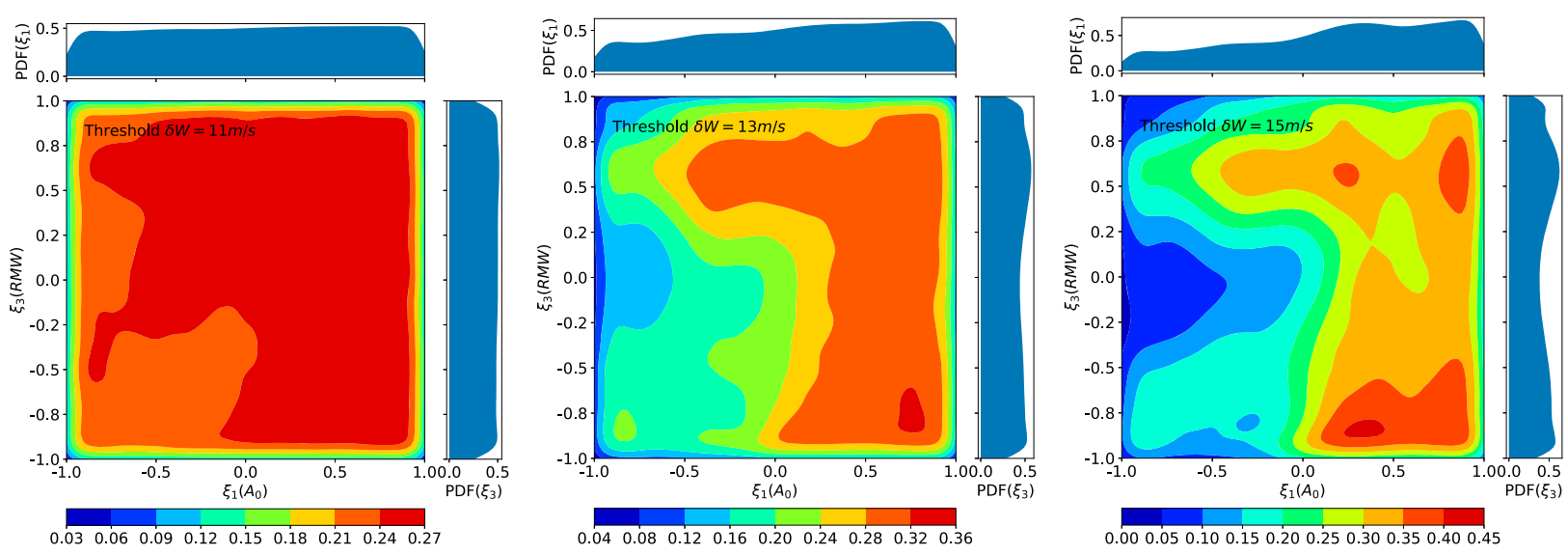

FIG. 14. Marginal and conditional probability distributions $p\left(\xi_{1}, \xi_{3} \mid \mathrm{RI}=1\right)$ of RI storm in the parameter space $\xi_{1}-\xi_{3}$ (associated with initial storm's intensity $A_{0}$ and RMW, respectively). The threshold is varied from 11 to $15 \mathrm{~m} \mathrm{~s}^{-1}$, as indicated. 


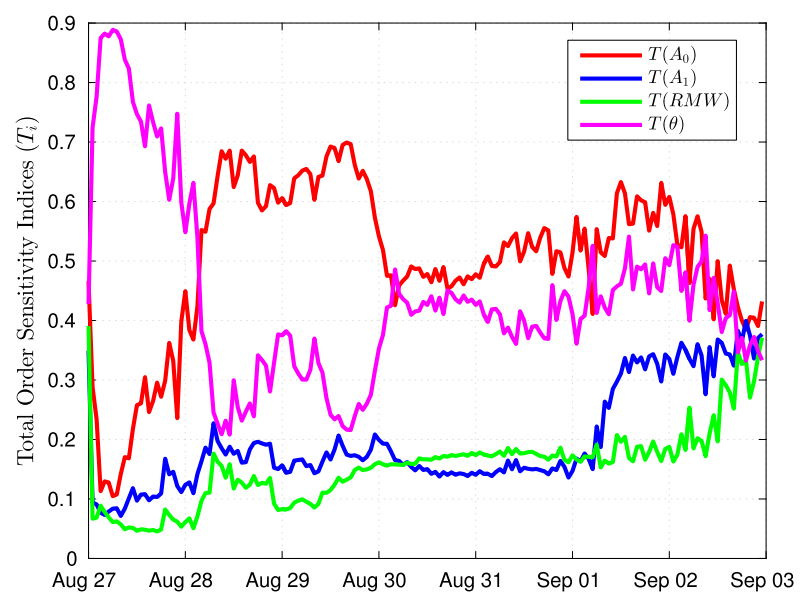

FIG. 15. Global sensitivity analysis of EOF-PC surrogate model of MWS evolution.

hand, the additional SKEBS random forcing leads to significantly wider storm-track variability (bottom panel in Fig. 16), which clearly indicates that the storm track is more sensitive to the environment
(SKEBS forcing) rather than internal properties of the storm.

Figure 17 examines more closely the SKEBS-forced realizations at each individual initial condition realization. It is seen that for almost all initial condition realizations, SKEBS forcing leads to dramatic divergence in the storm path, except for the first sample (the top-left panel in which SKEBS-forced storms move within the perturbed initial condition ensemble indicated by the red band). Meanwhile, some initial condition perturbations tend to favor one-sided bias (e.g., eastward bias in panels 2 and 10, and westward bias in panel 7), while others do not exhibit a trend (resulting storms can be on either side of the NHC best-estimated track). Overall, about twothirds of the 30 SKEBS-forced semiparametric simulations lead to storm tracks outside the perturbed initial condition ensemble boundary.

In addition, we have briefly explored the possibility of isolating the sensitivities associated with reducible (parametric) and irreducible (SKEBS) perturbations by applying the variance decomposition methodology
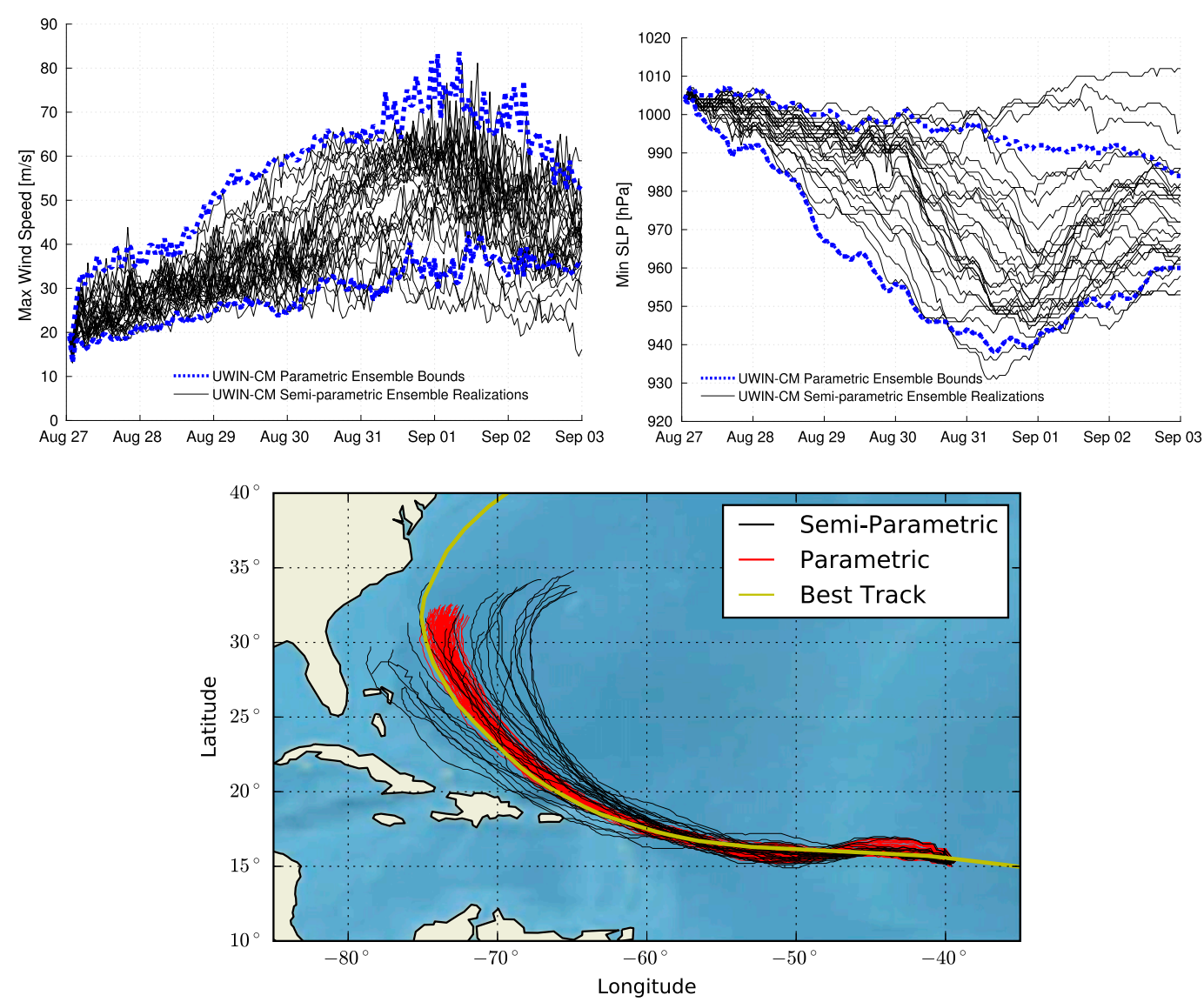

FIG. 16. Semiparametric realizations: (top left) MWS, (top right) MSLP, and (bottom) storm tracks. Also shown for comparison are the MWS MSLP bounds of the UWIN-CM initial condition ensemble, as well as the storm tracks of the initial condition ensemble. 

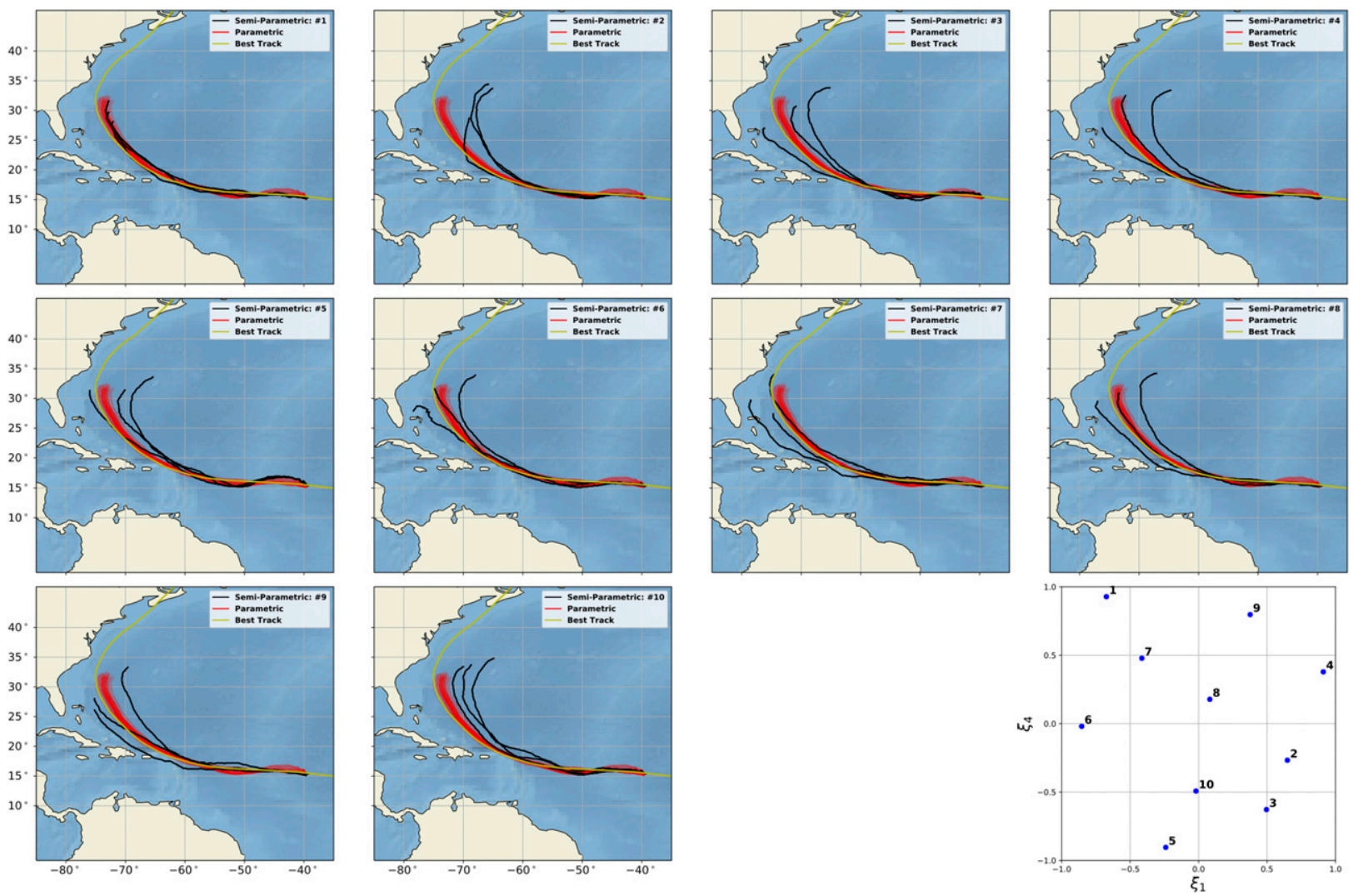

FIG. 17. Storm-track comparison: tracks from SKEBS simulations at each of the sample points in the $\xi_{1}-\xi_{4}$ domain (black), tracks from the initial condition simulation ensemble $\mathscr{P}_{\text {LHS }}^{80}$ (red), and the NHC best track estimate (yellow) and (bottom right) perturbation sample points.

recently introduced in Le Maître and Knio (2015), Le Maître et al. (2015) and Navarro Jimenez et al. (2016, 2017), which requires independent sampling of the reducible and irreducible perturbations. As outlined in Le Maître and Knio (2015), Le Maître et al. (2015), and Navarro Jimenez et al. (2016, 2017), independent samples of irreducible sources can be generated by acting on the seeds of the generators used to draw the samples, and this provides the means to extend variance decomposition concepts to irreducible uncertainty sources.

Along these lines, we generated a second random sample that accounts for both parameters and SKEBS forcing. Specifically, we considered two random seeds for the SKEBS perturbations, and for each value of the random seed, a 10-member Latin hypercube sampling of the mean wind intensity and rotation was considered. In other words, for each random seed, the two dominant initial storm parameters were randomly and independently sampled. This results in an additional 20-member ensemble (not shown for brevity). Examination of the results of this semiparametric sampling revealed trends that are similar to those observed in Fig. 16. Further extension of this strategy to perform a variance decomposition was unfortunately not possible because of a combination of several factors: 1) the limited Monte Carlo sampling of semiparametric UWIN-CM simulations that we could afford; 2) lack of repeatability of the UWIN-CM code in its parallel version, which prevents us from associating variability exclusively with SKEBS random seeds and/or initial condition perturbations; and 3) extreme sensitivity of storm track to global SKEBS perturbations, which raises fundamental questions concerning the significance of contrasting storm QoIs in the presence of large differences in storm tracks. Consequently, new capabilities are needed to overcome these hurdles, and these may be best established by considering smaller systems and tests involving individual components. The development of such capabilities is beyond the scope of the current study, and will be pursued elsewhere.

\section{Conclusions and discussion}

This study primarily aimed at analyzing Hurricane Earl's (2010) response to uncertainties in initial storm properties. Four uncertain parameters, namely, the 
azimuthally averaged wind speed, RMW, and asymmetry magnitude and azimuth, were used to encode internal uncertainties in the initial storm. The amplitudes of initial perturbations were based on characteristic uncertainties of the operational tropical cyclone observing systems (Landsea and Franklin 2013). The resulting initial perturbations were propagated through the UWIN-CM framework, which solves for time response of the entire atmosphere-wave-ocean coupled system. The coupled model simulations of Earl captured the observed intensity evolution during the first 5 days, but did not capture well its reintensification on 2 September. However, UWIN-CM produced near excellent prediction of Earl's track, which is an improvement over the current state of the art (Judt et al. 2016a; Smith et al. 2017). We focused on the time evolution of MWS, MSLP, and storm tracks with respect to different initial condition perturbations.

An 80-member UWIN-CM ensemble of initial condition perturbations was generated via LHS design in four-dimensional space, and a Bayesian KL analysis (Chowdhary and Najm 2016) was applied to examine uncertainties associated with each EOF mode. This EOF uncertainty analysis leads us to confidently adopt the EOFPC approach proposed in Li et al. (2016) for the purpose of building functional representations of time evolution of both MWS and MSLP. The agreement between UWIN-CM ensembles and PC predictions showed that our EOF-PC surrogate models, despite the finite truncation in EOF/PC expansions and regularization in solving the regression problem for PC expansion coefficients, effectively capture most of the UWIN-CM response variability, and are statistically consistent with Bayesian KL resampling results (see Figs. 7 and 10).

Our analysis showed that Earl's response (MWS and MSLP) variability increase rapidly over time, roughly along with the storm intensification until noon 31 August, indicating the complexity of storm RI dynamics. The RI classification criterion in Kaplan and DeMaria (2003) was adopted in this study. While this criterion seems to work well on raw UWIN-CM outputs, it is not suitable for either Bayesian KL or EOF-PC reconstructed responses, because of their inherent smoothing mechanism. Furthermore, using a prescribed RI classification threshold is obviously not a stable or robust measure, especially in light of the extremely noisy MWSs observed. To guard against the arbitrariness in adjusting the threshold parameter for RI prediction, we relied on the conditional probability distribution of initial random perturbations, given a storm undergoes RI. This conditional probability distribution measure remains stable and self-consistent across different RI thresholds. More importantly, this distribution measure partitions parameter space into subdomains with different likelihood of observing RI; for instance, increasing the initial storm strength and counterclockwise rotation of the storm were more likely to result in RI. In addition, this distribution measure also provides detailed insight on model sensitivities to different random parameters. Specifically, we find higher sensitivity to storm asymmetry azimuth on the first day of the simulation when the environmental shear was low, and higher sensitivity to azimuthally averaged maximum wind speed when the environmental shear increased significantly. This suggests that the uncertainty in initial vortex asymmetry may have greater impact on TC intensity in weakly sheared environments. However, further investigation and analysis to other TC cases is necessary to make more general conclusions.

Finally, we combined our initial condition perturbation scheme with the stochastic SKEBS forcing, and generated an additional 30-member UWIN-CM realization ensemble (30 independent SKEBS forcing realizations, 3 for each of the 10 LHS samples in canonical random variable space $\left.\xi_{1}-\xi_{4}\right)$. While either the initial condition perturbation or SKEBS forcing leads to considerably large (but comparable) variability in MWS and MSLP responses, the envelopes of these response variables were not further increased by combining the two perturbation mechanisms together. On the other hand, SKEBS forcing leads to wider storm-track span, suggesting sensitivity to the storm's environment.

The present work focused on investigating the impacts of the storm's initial configuration on its subsequent evolution, while keeping the initial large-scale environmental flow and oceanic conditions unperturbed. The PC analysis conducted here is inherently limited to the coupled model ensemble on which it is based. While the UWIN-CM prediction of Earl captured the observed intensity evolution during the first 5 days of the simulation, it did not capture well the reintensification of Earl on 2 September. Consequently, the PC analysis was unable to capture the later-stage variability of the storm. Furthermore, all conclusions are limited to the a priori assumptions made about the characteristic scale of uncertainty of the perturbed parameters. A more ambitious investigation program would have addressed both structural (irreducible) and parametric (reducible) sources of uncertainty, which have not been tested in this study, such as uncertainties in physical parameterizations; it would also have considered perturbing the environmental flow along with the oceanic conditions at the expense of increasing the size of the uncertain parameter space substantially, and thus the associated sampling requirements. The latter were already costly given the computational complexity of the coupled system. 
Another difficulty with assigning uncertainties to the large scale flows is: how best to perturb a field using the smallest number of uncertain variables. Thacker et al. (2012) and Iskandarani et al. (2016a) have successfully used empirical orthogonal function decomposition to perturb fields; however, the issue of identifying the relevant dynamical processes, in the ocean and atmosphere, whose uncertainties can potentially impact the hurricane forecast via EOFs, remains a nontrivial question.

In contrast to the initial condition uncertainties investigated here, the SKEBS stochastic representation had a large impact on the track and RI. One should note that the SKEBS perturbations were acting on the environmental flows and produced widely varying tracks and intensity responses. A perturbation of the environmental flows via EOFs could have resulted in large variability in hurricane track and intensification. Note, however, that these EOFs would have represented uncertainties in the large-scale flows at the initial time whereas SKEBS was developed to represent the impacts of stochastic uncertainties in the subgrid scale on the resolvable scales. The two approaches should not be seen as mutually exclusive but as complementary in attempting to quantify the impacts of input uncertainty and model uncertainty on the forecast. Thus, developing techniques to handle both types of uncertainties remains a necessity.

The present study involved the application of a PC methodology in a most complex setting and illustrated the benefits of building a surrogate that can suitably represent the functional relationship between uncertain inputs and model outputs. In the context of this study, the combination of regularized regression and reduced eigenspace projection techniques provide robust approach to surrogate construction. As has been observed in other settings ( $\mathrm{Li}$ et al. 2016; Sraj et al. 2016), the capabilities afforded by such approaches include the means to quantify and control the impact of regularization and truncation errors that inherently arise because of limited size ensembles and to QoIs that exhibit high-frequency oscillations. Once it is available, the surrogate can be readily exploited to conduct statistical analysis, determine the dominant contributors to model uncertainties, and assess the robustness of the sampling. Furthermore, it can be used to quantify the uncertainties in forecasting specific events, as was done in the present work, or in investigating the dynamical implications of various interacting processes, for example, in studying the impacts of shear height and depth on hurricane intensification as was done in Finocchio et al. (2016).

Acknowledgments. We thank three anonymous reviewers who helped improved this manuscript. This research was made possible in part by a grant from the
Gulf of Mexico Research Initiative, and by the U.S. Department of Energy (DOE), Office of Science, Office of Advanced Scientific Computing Research, under Award DE-SC0008789, NASA Ocean Vector Wind Science Team Grant NNX14AM78G, and by the National Science Foundation under Grant 1639722 (an EarthCubefunded project). Computations were performed using the resources of the National Energy Research Scientific Computing Center, a DOE Office of Science user facility supported by the Office of Science of the U.S. Department of Energy under Contract DE-AC02-05CH11231. Data are publicly available (https://data.gulfresearchinitiative.org; Gulf of Mexico Research Initiative Information and Data Cooperative 2017).

\section{APPENDIX A}

\section{Coupled Model Description and Configuration}

The coupled modeling system used in this study is the Unified Wave Interface-Coupled Model (UWIN-CM; Chen et al. 2013; Chen and Curcic 2016). It is developed using physically based air-sea coupling through surface waves and designed as a multimodel system that is compatible with the next-generation coupled atmospherewave-ocean-land-sea ice Earth system models. The coupling interface explicitly resolves wind-wave and wave-current interactions and exchanges momentum between components in a conservative and physically consistent way. The model has been used to study the role of Stokes drift in surface transport (Curcic et al. 2016), and the impacts of (i) coupling on boundary layer structure (Zhu et al. 2016) in Hurricane Isaac (2012), as well as (ii) atmospheric forcing on the transport in Gulf of Mexico on diurnal and seasonal scales (Judt et al. 2016b). In this study, the fully coupled model components consist of atmosphere, surface waves, and ocean circulation models. The following model description is largely adapted from Chen and Curcic (2016).

\section{a. Atmosphere model}

The atmosphere model used in UWIN-CM is the Weather Research and Forecasting (WRF) Model v3.7.1 with Advanced Research WRF (ARW) dynamical core (Skamarock et al. 2008). WRF is a nonhydrostatic atmospheric model with many model physics options and a storm-following, moving nest capability for hurricane forecasting.

The surface layer physics are based on the MoninObukhov theory and boundary layer mixing is parameterized using the Yonsei University scheme (YSU; Hong et al. 2006). The cloud microphysics is the single-moment 6-class microphysics scheme (WSM6; Hong et al. 2004), 
which is used on the inner nested domains without cumulus parameterization. The Kain-Fritsch cumulus parameterization is used only on the $12-\mathrm{km}$ parent domain (Kain and Fritsch 1993) in addition to WSM6. In UWIN-CM, WRF passes surface wind vectors and air density to the wave model, and receives surface stress vector from the wave model, and sea surface temperature (SST) and currents from the ocean circulation model. All fields that are exchanged between WRF and other coupled components are directly interpolated to and from each nest.

\section{b. Ocean surface wave model}

The ocean surface wave model in UWIN-CM is the University of Miami Wave Model (UMWM) v2 (Donelan et al. 2012). It is a third-generation spectral ocean wave model that predicts wave energy spectra and the atmosphere and ocean momentum fluxes based on winds and ocean currents. In UWIN-CM, UMWM passes air-side and water-side vector stresses to atmosphere and ocean circulation models, respectively. UMWM also takes into account Eulerian ocean currents that act to advect and refract surface wave energy.

\section{c. Ocean circulation model}

The ocean circulation model in UWIN-CM is the Hybrid Coordinate Ocean Model (HYCOM) v2.2 (Wallcraft et al. 2009). It is a three-dimensional hydrostatic ocean model with a hybrid vertical coordinate: $z$ level in shallow water, terrain-following coordinate in intermediate water, and isopycnal (constant density) in deep water. In this study, we use the nonlocal $K$-profile vertical mixing scheme by Large et al. (1994). Full tidal forcing from both solar and lunar tide components are enabled in our simulations. In UWIN-CM, HYCOM receives surface stress based on wave dissipation computed by the wave model, and turbulent and radiative heat fluxes from the atmosphere model.

\section{d. Atmosphere-wave-ocean coupling}

The UWIN handles the coupling between model components. It contains the coupling physics and a common exchange grid that is used for interpolation and calculation of air-sea exchange fields from the component models. WRF passes the wind profile and air density to the wave model and radiative and heat fluxes and precipitation rate to the ocean circulation model. UMWM passes vectorial atmosphere stress to the atmosphere model and vectorial ocean stress to the ocean circulation model. HYCOM passes sea surface temperature (SST) to the atmosphere model and surface current field and water density to the wave model. All fields are interpolated to the exchange grid and are collocated in discrete time. In the current model configuration, fields

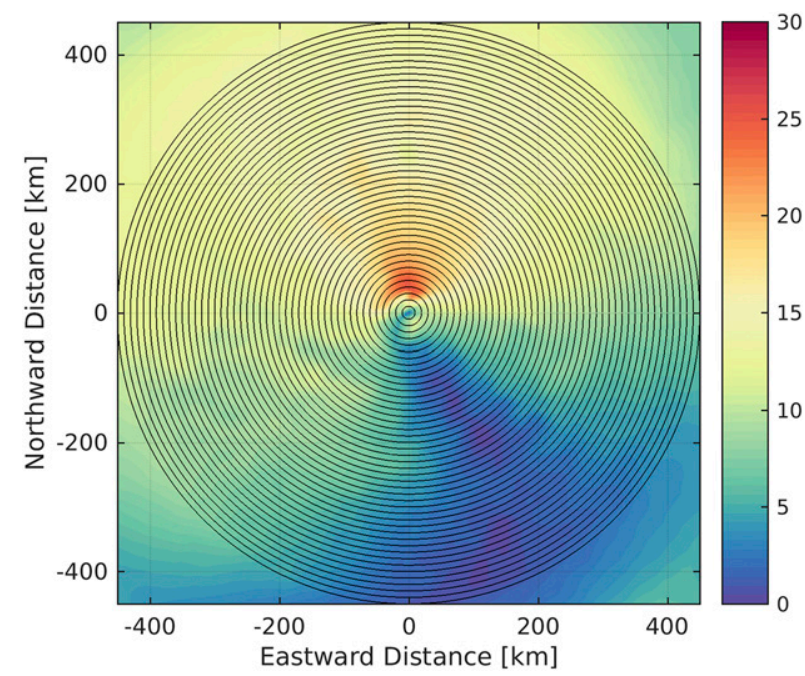

FIG. B1. Discretization of wind field into 10-km-width circular annuli.

between all components are exchanged every $60 \mathrm{~s}$. The interpolation of fields between different model grids, and parallel execution of the coupler is implemented using the Earth System Modeling Framework (ESMF; Hill et al. 2004).

\section{APPENDIX B}

\section{Hurricane Earl Perturbation Formulation}

The initial wind field of Hurricane Earl is shown in Fig. 1. For clarity, we decompose the initial azimuthal wind $w$ as follows:

$$
\begin{aligned}
w(r, \beta)= & A_{0}(r)+A_{1}(r) \cos \left[\beta-\beta_{1}(r)\right] \\
& +\operatorname{Res}(r, \beta) \forall r \in[0, R],
\end{aligned}
$$

where $(r, \beta)$ is a cylindrical coordinate system centered on the storm, and $R=450 \mathrm{~km}$ denotes the boundary of the storm. The terms $A_{0}(r)$ and $A_{1}(r)$ are wind amplitudes of wavenumber- 0 and -1 components, respectively, at radius $r ; \beta_{1}(r)$ is the wavenumber- 1 asymmetry azimuth (further discussed below); and $\operatorname{Res}(r, \beta)$ denotes the residual wind field.

To calculate the radial profiles of $A_{0}(r)$ and $\left[A_{1}(r), \beta_{1}(r)\right]$ in Eq. (B1), we first discretize the wind field into $\Delta r=10-\mathrm{km}$-wide annuli along the radial direction, as shown in Fig. B1.Each annulus is indexed by an integer $i(i=1,2, \ldots, N$ with increasing radius) and bounded by the radius of its inner and outer boundaries (i.e., $r_{i-1}$ and $r_{i}$, respectively, with $r_{0}=0$ ). The wavenumber- 0 component is simply the averaged wind speed in each annulus, that is, 
$A_{0}(r)=\frac{1}{\pi\left(r_{i}^{2}-r_{i-1}^{2}\right)} \int_{r_{i-1}}^{r_{i}} \int_{0}^{2 \pi} w(r, \beta) r d r d \beta \forall r \in\left[r_{i-1}, r_{i}\right)$
Once $A_{0}(r)$ is calculated, $\left[A_{1}(r), \beta_{1}(r)\right]$ are simply the results of azimuthal least squares fitting of $w(r, \beta)-A_{0}(r)$ onto $\cos \left[\beta-\beta_{1}(r)\right]$ function in each of the annulus, that is,

$$
A_{1}(r), \beta_{1}(r)=\underset{A_{1}, \beta_{1}}{\operatorname{argmin}} \int_{r_{i-1}}^{r_{i}} \int_{0}^{2 \pi}\left\{w(r, \beta)-A_{0}(r)-A_{1}(r) \cos \left[\beta-\beta_{1}(r)\right]\right\}^{2} r d r d \beta \forall r \in\left[r_{i-1}, r_{i}\right) .
$$

Finally, the residue $\operatorname{Res}(r, \beta)$ is simply

$$
\begin{aligned}
\operatorname{Res}(r, \beta)= & w(r, \beta)-A_{0}(r) \\
& -A_{1}(r) \cos \left[\beta-\beta_{1}(r)\right] \forall r \in[0,450] .
\end{aligned}
$$

To design parametric perturbations of the initial wind field, we focus on three aspects of the storm: the storm strength [characterized by $A_{0}(r)$ and $A_{1}(r)$ ], storm core size (characterized by RMW), and storm rotation. We first define RMW as

$$
r_{\mathrm{RMW}}=\underset{r \leq 300 \mathrm{~km}}{\operatorname{argmax}} A_{0}(r) .
$$

The intensity and asymmetry amplitude [either $A_{0}(r)$ or $\left.A_{1}(r)\right]$ is perturbed (up to $\pm 20 \%$ ) via

$$
A_{0,1}^{I}(r)=\left\{\begin{array}{ll}
A_{0,1}(r) \times\left(1+0.2 \times \xi_{1,2}\right) & \text { for } \quad r \leq r_{\mathrm{RMW}} \\
A_{0,1}(r)+0.2 \times \xi_{1,2} \times A_{0,1}\left(r_{\mathrm{RMW}}\right) \frac{R-r}{R-r_{\mathrm{RMW}}} & \text { for } \quad r_{\mathrm{RMW}}<r \leq R
\end{array},\right.
$$

where the superscript $I$ indicate the first stage of the perturbation. Here $\xi_{i} \in[-1,1]$ denote uniformly distributed random parameters $\left(\xi_{1}, \xi_{2}\right.$ corresponds to $A_{0}, A_{1}$, respectively). The second part of Eq. (B6) is used to ensure smooth transition across the storm boundary at $R=450 \mathrm{~km}$.
The second perturbation stage concerns the storm size (RMW). Because RMW is defined from the $A_{0}(r)$ profile, perturbing RMW $\left[r_{\mathrm{RMW}}^{*}=\left(1+0.2 \times \xi_{3}\right) r_{\mathrm{RMW}}\right]$ leads to stretching/shrinking of the $A_{0}^{I}(r)$ profile as follows:

$$
A_{0}^{I I}(r)=\left\{\begin{array}{ll}
A_{0}\left(\frac{r}{1+r_{\mathrm{RMW}}}\right) & \text { for } \quad r \leq r_{\mathrm{RMW}}^{*} \\
A_{0}\left[R-\frac{R-r_{\mathrm{RMW}}}{R-r_{\mathrm{RMW}}^{*}}(R-r)\right] & \text { for } \quad r_{\mathrm{RMW}}^{*}<r \leq R
\end{array} .\right.
$$

Again, the second row in the above equation is to ensure smooth transition across the storm boundary. The perturbed wind speed field thus reads as

$$
\begin{aligned}
w^{I I}(r, \beta)= & A_{0}^{I I}(r)+A_{1}^{I}(r) \cos \left[\beta-\beta_{1}(r)\right] \\
& +\operatorname{Res}(r, \beta) \forall r \in[0, R] .
\end{aligned}
$$

It is noted that unlike perturbing the storm strength and size where only the two dominant wave components $A_{0}(r)$ and $A_{1}(r)$ are perturbed, storm rotation is applied to the whole wind field. Given a rotating angle $\left[-\pi / 2 \leq \alpha_{0}=(\pi / 2) \xi_{4} \leq \pi / 2\right]$, the storm is rotated according to the following linearly decaying angle profile at different locations:

$$
\alpha(r)=\frac{R-r}{R} \alpha_{0}=\frac{R-r}{R} \frac{\pi}{2} \xi_{4} .
$$

The above linear decay profile of the rotation angle is designed to ensure smooth wind field transition across the storm boundary. To rotate the entire wind field, the following rotation operations are required:

1) Rotate the wind speed (scalar) field:

This is done by a linear transformation of the spatial coordinates. Let $(x, y)$ be the original spatial grid coordinate and $r=\sqrt{x^{2}+y^{2}}$ be the distance between the storm center, and $(x, y)$, the new grid coordinate after rotating an angle of $\alpha(r)$ is given by 

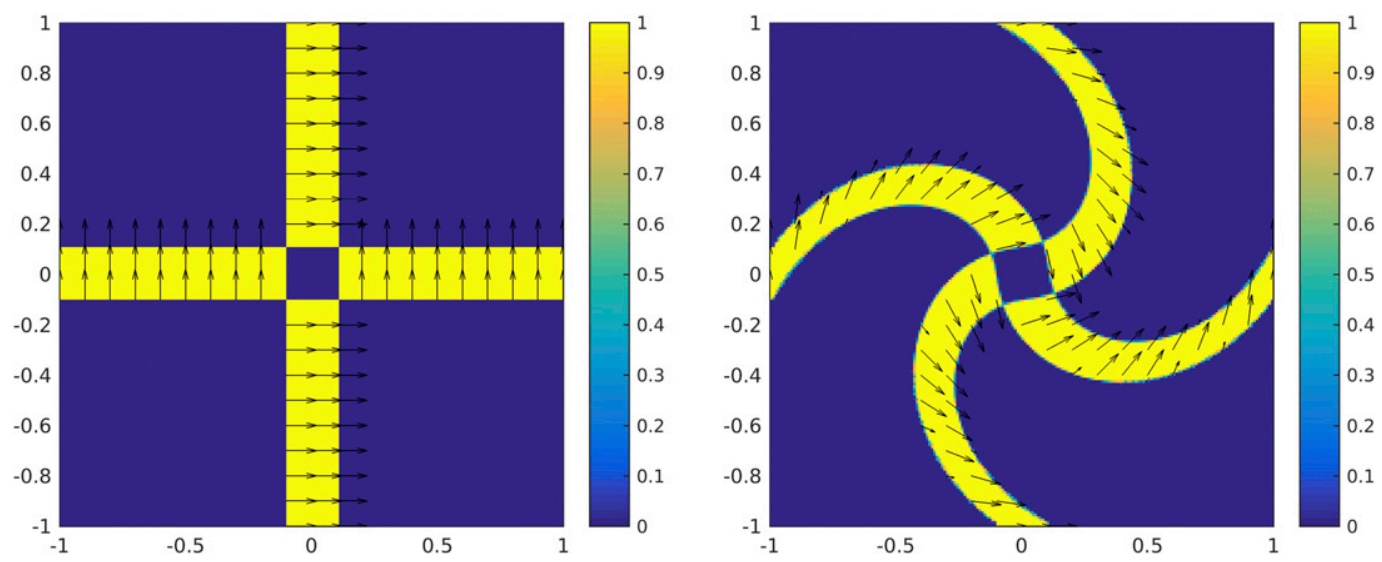

FIG. B2. Illustration of vector field rotation: (left) original field and (right) rotated field. The color map indicates strength (in terms of vector norm) of the field.

$$
\left[\begin{array}{l}
x^{*} \\
y^{*}
\end{array}\right]=\left[\begin{array}{cc}
\cos [\alpha(r)] & -\sin [\alpha(r)] \\
\sin [\alpha(r)] & \cos [\alpha(r)]
\end{array}\right]\left[\begin{array}{l}
x \\
y
\end{array}\right]
$$

By mapping the scalar function values at the original grid to rotated grid, we end up with a rotated scalar field. Specifically, we have

$$
\begin{aligned}
\hat{f}\left(x^{*}, y^{*}\right) & =f(x, y) \\
& =f\left(\left[\begin{array}{cc}
\cos [\alpha(r)] & -\sin [\alpha(r)] \\
\sin [\alpha(r)] & \cos [\alpha(r)]
\end{array}\right]^{-1}\left[\begin{array}{c}
x^{*} \\
y^{*}
\end{array}\right]\right),
\end{aligned}
$$

where $f(x, y)$ is a generic scalar function.

2) Rotate the wind velocity vectors:

This is done by first rotating the velocity component fields $U$ and $V$, respectively, from the previous step, and then adding the rotation angle $\alpha(r)$ to the velocity orientation angle, that is,

$$
\beta\left(x^{*}, y^{*}\right)=\alpha(r)+\arctan \left(\frac{\hat{V}}{\hat{U}}\right),
$$

where $\beta\left(x^{*}, y^{*}\right)$ denotes the new velocity orientation angle after rotation. Figure B2 illustrates the rotation mechanism discussed above.

\section{REFERENCES}

Alexanderian, A., J. Winokur, I. Sraj, A. Srinivasan, M. Iskandarani, W. C. Thacker, and O. M. Knio, 2012: Global sensitivity analysis in an ocean general circulation model: A sparse spectral projection approach. Comput. Geosci., 16, 757-778, https:// doi.org/10.1007/s10596-012-9286-2.

Alves, J.-H. G., S. Stripling, A. Chawla, H. Tolman, and A. van der Westhuysen, 2015: Operational wave guidance at the U.S.
National Weather Service during Tropical/Post-Tropical Storm Sandy, October 2012. Mon. Wea. Rev., 143, 1687-1702, https://doi.org/10.1175/MWR-D-14-00143.1.

Anthes, R. A., and S. W. Chang, 1978: Response of the hurricane boundary layer to changes of sea surface temperature in a numerical model. J. Atmos. Sci., 35, 1240-1255, https://doi.org/ 10.1175/1520-0469(1978)035<1240:ROTHBL >2.0.CO;2.

Brown, B. R., and G. J. Hakim, 2013: Variability and predictability of a three-dimensional hurricane in statistical equilibrium. J. Atmos. Sci., 70, 1806-1820, https://doi.org/10.1175/JAS-D-12-0112.1.

Cangialosi, J. P., 2011: Tropical cyclone report: Hurricane Earl (25 August-4 September 2010). Tech. Rep. AL072010, National Hurricane Center, 29 pp., https://www.nhc.noaa.gov/ data/tcr/AL072010_Earl.pdf.

Chen, S. S., and M. Curcic, 2016: Ocean surface waves in Hurricane Ike (2008) and Superstorm Sandy (2012): Coupled model predictions and observations. Ocean Modell., 103, 161-176, https://doi.org/10.1016/j.ocemod.2015.08.005.

—, W. Zhao, M. A. Donelan, J. F. Price, and E. J. Walsh, 2007: The CBLAST-Hurricane program and the next-generation fully coupled atmosphere-wave-ocean models for hurricane research and prediction. Bull. Amer. Meteor. Soc., 88, 311-317, https://doi.org/10.1175/BAMS-88-3-311.

,,,--- and H. L. Tolman, 2013: Directional wind-wave coupling in fully coupled atmosphere-wave-ocean models: Results from CBLAST-Hurricane. J. Atmos. Sci., 70, 31983215, https://doi.org/10.1175/JAS-D-12-0157.1.

Chowdhary, K., and H. N. Najm, 2016: Bayesian estimation of Karhunen-Loève expansions: A random subspace approach. J. Comput. Phys., 319, 280-293, https://doi.org/ 10.1016/j.jcp.2016.02.056.

Conrad, P. R., and Y. M. Marzouk, 2013: Adaptive Smolyak pseudospectral approximations. SIAM J. Sci. Comput., 35, A2643-A2670, https://doi.org/10.1137/120890715.

Curcic, M., S. S. Chen, and T. M. Özgökmen, 2016: Hurricaneinduced ocean waves and Stokes drift and their impacts on surface transport and dispersion in the Gulf of Mexico. Geophys. Res. Lett., 43, 2773-2781, https://doi.org/10.1002/ 2015 GL067619.

Davidson, N. E., and Coauthors, 2014: ACCESS-TC: Vortex specification, 4DVAR initialization, verification, and structure diagnostics. Mon. Wea. Rev., 142, 1265-1289, https:// doi.org/10.1175/MWR-D-13-00062.1. 
Dickey, T., and Coauthors, 1998: Upper-ocean temperature response to Hurricane Felix as measured by the Bermuda testbed mooring. Mon. Wea. Rev., 126, 1195-1201, https://doi.org/ 10.1175/1520-0493(1998)126<1195:UOTRTH > 2.0.CO;2.

Donelan, M. A., M. Curcic, S. S. Chen, and A. K. Magnusson, 2012: Modeling waves and wind stress. J. Geophys. Res., 117, C00J23, https://doi.org/10.1029/2011JC007787.

Finocchio, P. M., S. J. Majumdar, D. S. Nolan, and M. Iskandarani, 2016: Idealized tropical cyclone responses to the height and depth of environmental vertical wind shear. Mon. Wea. Rev., 144, 2155-2175, https://doi.org/10.1175/MWR-D-15-0320.1.

Funakoshi, Y., S. C. Hagen, and P. Bacopoulos, 2008: Coupling of hydrodynamic and wave models: Case study for Hurricane Floyd (1999) hindcast. J. Waterw. Port Coastal Ocean Eng., 134, 321-335, https://doi.org/10.1061/(ASCE)0733-950X(2008)134: 6(321).

Ghanem, R. G., and P. D. Spanos, 1991: Stochastic Finite Elements: A Spectral Approach. Springer-Verlag, 214 pp., https://doi.org/ 10.1007/978-1-4612-3094-6.

Gulf of Mexico Research Initiative Information and Data Cooperative, 2017: 110 UWIM-CM simulations of Hurricane Earl, August 27 to September 2, 2010: (Atlantic, Caribbean, Gulf of Mexico) with random hurricane parameter inputs. Consortium for Advanced Research on Transport of Hydrocarbon in the Environment II (CARTHE II), accessed 5 June 2018, https://doi.org/10.7266/N7JS9P2W.

Hakim, G. J., 2013: The variability and predictability of axisymmetric hurricanes in statistical equilibrium. J. Atmos. Sci., 70, 993-1005, https://doi.org/10.1175/JAS-D-12-0188.1.

Hendricks, E. A., M. S. Peng, and T. Li, 2013: Evaluation of multiple dynamic initialization schemes for tropical cyclone prediction. Mon. Wea. Rev., 141, 4028-4048, https://doi.org/ 10.1175/MWR-D-12-00329.1.

Hill, C., C. DeLuca, V. Balaji, M. Suarez, and A. da Silva, 2004: Architecture of the earth system modeling framework. Comput. Sci. Eng., 6, 18-28, https://doi.org/10.1109/ MCISE.2004.1255817.

Homma, T., and A. Saltelli, 1996: Importance measures in global sensitivity analysis of nonlinear models. Reliab. Eng. Syst. Saf., 52, 1-17, https://doi.org/10.1016/0951-8320(96)00002-6.

Hong, S.-Y., J. Dudhia, and S.-H. Chen, 2004: A revised approach to ice microphysical processes for the bulk parameterization of clouds and precipitation. Mon. Wea. Rev., 132, 103-120, https://doi.org/10.1175/1520-0493(2004)132<0103: ARATIM $>2.0 . \mathrm{CO} ; 2$.

—, Y. Noh, and J. Dudhia, 2006: A new vertical diffusion package with an explicit treatment of entrainment processes. Mon. Wea. Rev., 134, 2318-2341, https://doi.org/10.1175/ MWR3199.1.

Hope, M. E., and Coauthors, 2013: Hindcast and validation of Hurricane Ike (2008) waves, forerunner, and storm surge. J. Geophys. Res. Oceans, 118, 4424-4460, https://doi.org/10.1002/ jgrc.20314.

Hsu, S., 2013: Storm surges in New York during Hurricane Sandy in 2012: A verification of the wind-stress tide relation. Bound.-Layer Meteor., 148, 593-598, https://doi.org/ 10.1007/s10546-013-9830-1.

— J. J. Mrymes III, and Z. Yan, 1997: A simplified hydrodynamic formula for estimating the wind-driven flooding in the Lake Pontchartrain-Amite River basin. Natl. Wea. Dig., 21 (4), 18-22.

Iskandarani, M., M. Le Hénaff, W. C. Thacker, A. Srinivasan, and O. M. Knio, 2016a: Quantifying uncertainty in Gulf of
Mexico forecasts stemming from uncertain initial conditions. J. Geophys. Res. Oceans, 121, 4819-4832, https://doi.org/10.1002/ 2015JC011573.

_ S. Wang, A. Srinivasan, W. Thacker, J. Winokur, and O. Knio, 2016b: An overview of uncertainty quantification techniques with application to oceanic and oil-spill simulations. J. Geophys. Res. Oceans, 121, 2789-2808, https://doi.org/ 10.1002/2015JC011366.

Janjić, T., and Coauthors, 2018: On the representation error in data assimilation. Quart. J. Roy. Meteor. Soc., 144, 1257-1278, https://doi.org/10.1002/qj.3130.

Judt, F., and S. S. Chen, 2016: Predictability and dynamics of tropical cyclone rapid intensification deduced from highresolution stochastic ensembles. Mon. Wea. Rev., 144, 43954420, https://doi.org/10.1175/MWR-D-15-0413.1.

—_ — _ , and J. Berner, 2016a: Predictability of tropical cyclone intensity: Scale-dependent forecast error growth in high-resolution stochastic kinetic-energy backscatter ensembles. Quart. J. Roy. Meteor. Soc., 142, 43-57, https:// doi.org/10.1002/qj.2626.

, — , and M. Curcic, 2016b: Atmospheric forcing of the upper ocean transport in the Gulf of Mexico: From seasonal to diurnal scales. J. Geophys. Res. Oceans, 121, 4416-4433, https:// doi.org/10.1002/2015JC011555.

Kain, J. S., and J. M. Fritsch, 1993: Convective parameterization for mesoscale models: The Kain-Fritsch scheme. The Representation of Cumulus Convection in Numerical Models, Meteor. Monogr., No. 24, Amer. Meteor. Soc., 165-170.

Kaplan, J., and M. DeMaria, 2003: Large-scale characteristics of rapidly intensifying tropical cyclones in the North Atlantic basin. Wea. Forecasting, 18, 1093-1108, https://doi.org/10.1175/ 1520-0434(2003)018<1093:LCORIT>2.0.CO;2.

,$- \ldots$, and J. A. Knaff, 2010: A revised tropical cyclone rapid intensification index for the Atlantic and eastern North Pacific basins. Wea. Forecasting, 25, 220-241, https://doi.org/10.1175/ 2009WAF2222280.1.

Landsea, C. W., and J. L. Franklin, 2013: Atlantic hurricane database uncertainty and presentation of a new database format. Mon. Wea. Rev., 141, 3576-3592, https://doi.org/10.1175/ MWR-D-12-00254.1.

Large, W. G., J. C. McWilliams, and S. C. Doney, 1994: Oceanic vertical mixing: A review and a model with a nonlocal boundary layer parameterization. Rev. Geophys., 32, 363-403, https://doi.org/10.1029/94RG01872.

Latif, M., N. Keenlyside, and J. Bader, 2007: Tropical sea surface temperature, vertical wind shear, and hurricane development. Geophys. Res. Lett., 34, L01710, https://doi.org/10.1029/ 2006GL027969.

Lee, C.-Y., and S. S. Chen, 2014: Stable boundary layer and its impact on tropical cyclone structure in a coupled atmosphereocean model. Mon. Wea. Rev., 142, 1927-1944, https://doi.org/ 10.1175/MWR-D-13-00122.1.

Le Maître, O. P., and O. M. Knio, 2010: Spectral Methods for Uncertainty Quantification: With Applications to Computational Fluid Dynamics. Springer, 536 pp., https://doi.org/10.1007/97890-481-3520-2.

- , and -2015 : PC analysis of stochastic differential equations driven by Wiener noise. Reliab. Eng. Syst. Saf., 135, 107124, https://doi.org/10.1016/j.ress.2014.11.002.

- — , H. N. Najm, and R. G. Ghanem, 2001: A stochastic projection method for fluid flow: I. Basic formulation. J. Comput. Phys., 173, 481-511, https://doi.org/10.1006/ jcph.2001.6889. 
- M. T. Reagan, H. N. Najm, R. G. Ghanem, and O. M. Knio, 2002: A stochastic projection method for fluid flow: II. Random process. J. Comput. Phys., 181, 9-44, https://doi.org/ 10.1006/jcph.2002.7104.

_- O. M. Knio, and A. Moraes, 2015: Variance decomposition in stochastic simulators. J. Chem. Phys., 142, 244115, https:// doi.org/10.1063/1.4922922.

Letson, D., D. S. Sutter, and J. K. Lazo, 2007: Economic value of hurricane forecasts: An overview and research needs. Nat. Hazards Rev., 8, 78-86, https://doi.org/10.1061/(ASCE)15276988(2007)8:3(78).

Li, G., M. Iskandarani, M. Le Hénaff, J. Winokur, O. P. Le Maître, and O. M. Knio, 2016: Quantifying initial and wind forcing uncertainties in the Gulf of Mexico. Comput. Geosci., 20, 1133-1153, https://doi.org/10.1007/s10596-016-9581-4.

Mattern, J., K. Fennel, and M. Dowd, 2012: Estimating timedependent parameters for a biological ocean model using an emulator approach. J. Mar. Syst., 96-97, 32-47, https://doi.org/ 10.1016/j.jmarsys.2012.01.015.

McKay, M. D., R. J. Beckman, and W. J. Conover, 1979: Comparison of three methods for selecting values of input variables in the analysis of output from a computer code. Technometrics, 21 (2), 239-245.

Montgomery, M. T., J. A. Zhang, and R. K. Smith, 2014: An analysis of the observed low-level structure of rapidly intensifying and mature Hurricane Earl (2010). Quart. J. Roy. Meteor. Soc., 140, 2132-2146, https://doi.org/10.1002/ qj.2283.

Mycek, P., and Coauthors, 2017: A resilient domain decomposition polynomial chaos solver for uncertain elliptic PDEs. Comput. Phys. Commun., 216, 18-34, https://doi.org/ 10.1016/j.cpc.2017.02.015.

Navarro Jimenez, M., O. Le Maître, and O. Knio, 2016: Global sensitivity analysis in stochastic simulators of uncertain reaction networks. J. Chem. Phys., 145, 244106, https://doi.org/ 10.1063/1.4971797.

,-- , and -2017 : Nonintrusive polynomial chaos expansions for sensitivity analysis in stochastic differential equations. SIAM ASA J. Uncertainty Quantif., 5, 378-402, https://doi.org/10.1137/16M1061989.

Nolan, D. S., R. Atlas, K. T. Bhatia, and L. R. Bucci, 2013: Development and validation of a hurricane nature run using the joint OSSE nature run and the WRF model. J. Adv. Model. Earth Syst., 5, 382-405, https://doi.org/ 10.1002/jame.20031.

Peng, J., J. Hampton, and A. Doostan, 2014: A weighted l1-minimization approach for sparse polynomial chaos expansions. J. Comput. Phys., 267, 92-111, https://doi.org/ 10.1016/j.jcp.2014.02.024.

Rogers, R. F., P. D. Reasor, and J. A. Zhang, 2015: Multiscale structure and evolution of Hurricane Earl (2010) during rapid intensification. Mon. Wea. Rev., 143, 536-562, https://doi.org/ 10.1175/MWR-D-14-00175.1.

Rosenfeld, D., A. Khain, B. Lynn, and W. Woodley, 2007: Simulation of hurricane response to suppression of warm rain by sub-micron aerosols. Atmos. Chem. Phys., 7, 3411-3424, https://doi.org/10.5194/acp-7-3411-2007.

Senn, H. V., and H. Hiser, 1959: On the origin of hurricane spiral rain bands. J. Meteor., 16, 419-426, https://doi.org/10.1175/ 1520-0469(1959)016<0419:OTOOHS >2.0.CO;2.

Singh, V., A. Routray, S. Mallick, J. P. George, and E. N. Rajagopal, 2016: An effort to improve track and intensity prediction of tropical cyclones through vortex initialization in
NCUM-global model. Proc. SPIE, 9882, 988210, https:// doi.org/10.1117/12.2223431.

Sippel, J. A., and F. Zhang, 2010: Factors affecting the predictability of Hurricane Humberto (2007). J. Atmos. Sci., 67, 1759-1778, https://doi.org/10.1175/2010JAS3172.1.

Siripatana, A., T. Mayo, I. Sraj, O. Knio, C. Dawson, O. Le Maître, and I. Hoteit, 2017: Assessing an ensemble Kalman filter inference of Manning's $n$ coefficient of an idealized tidal inlet against a polynomial chaos-based MCMC. Ocean Dyn., 67, 1067-1094, https://doi.org/10.1007/s10236-0171074-z.

Skamarock, W. C., and Coauthors, 2008: A description of the Advanced Research WRF version 3. NCAR Tech. Note NCAR/TN-475+STR, 113 pp., https://doi.org/10.5065/ D68S4MVH.

Smith, R. K., J. A. Zhang, and M. T. Montgomery, 2017: The dynamics of intensification in a Hurricane Weather Research and Forecasting simulation of Hurricane Earl (2010). Quart. J. Roy. Meteor. Soc., 143, 293-308, https://doi.org/10.1002/ qj. 2922.

Smith, T. A., and Coauthors, 2013: Ocean-wave coupled modeling in COAMPS-TC: A study of Hurricane Ivan (2004). Ocean Modell., 69, 181-194, https://doi.org/10.1016/ j.ocemod.2013.06.003.

Sobol, I., 1993: Sensitivity estimates for nonlinear mathematical models. Math. Model. Comput. Exp., 1, 407-414.

Sraj, I., and Coauthors, 2013: Bayesian inference of drag parameters using AXBT data from Typhoon Fanapi. Mon. Wea. Rev., 141, 2347-2367, https://doi.org/10.1175/MWR-D-12-00228.1.

, M. Iskandarani, W. C. Thacker, A. Srinivasan, and O. Knio, 2014: Drag parameter estimation using gradients and Hessian from a polynomial chaos model surrogate. Mon. Wea. Rev., 142, 933-941, https://doi.org/10.1175/MWR-D13-00087.1.

— S. E. Zedler, O. M. Knio, C. S. Jackson, and I. Hoteit, 2016: Polynomial chaos-based Bayesian inference of $k$-profile parameterization in a general circulation model of the tropical Pacific. Mon. Wea. Rev., 144, 4621-4640, https://doi.org/10.1175/ MWR-D-15-0394.1.

Stevenson, S. N., K. L. Corbosiero, and J. Molinari, 2014: The convective evolution and rapid intensification of Hurricane Earl (2010). Mon. Wea. Rev., 142, 4364-4380, https://doi.org/ 10.1175/MWR-D-14-00078.1.

Stone, G. W., J. M. Grymes III, J. R. Dingler, and D. A. Pepper, 1997: Overview and significance of hurricanes on the Louisiana coast, U.S.A. J. Coastal Res., 13 (3), 656-669.

Thacker, W. C., A. Srinivasan, M. Iskandarani, O. M. Knio, and M. Le Hénaff, 2012: Propagating boundary uncertainties using polynomial expansions. Ocean Modell., 43-44, 52-63, https:// doi.org/10.1016/j.ocemod.2011.11.011.

Uhlhorn, E. W., and D. S. Nolan, 2012: Observational undersampling in tropical cyclones and implications for estimated intensity. Mon. Wea. Rev., 140, 825-840, https://doi.org/10.1175/ MWR-D-11-00073.1.

Wallcraft, A. J., E. J. Metzger, and S. N. Carroll, 2009: Software design description for the HYbrid Coordinate Ocean Model (HYCOM), version 2.2. Tech. Rep. NRL/MR/7320—09-9166, NRL, Stennis Space Center, MS, 157 pp., http://www.dtic.mil/ dtic/tr/fulltext/u2/a494779.pdf.

Walsh, E., and Coauthors, 2002: Hurricane directional wave spectrum spatial variation at landfall. J. Phys. Oceanogr., 32, 1667-1684, https://doi.org/10.1175/1520-0485(2002)032<1667: HDWSSV $>2.0 . \mathrm{CO} ; 2$. 
Winokur, J., P. Conrad, I. Sraj, O. Knio, A. Srinivasan, W. C. Thacker, Y. Marzouk, and M. Iskandarani, 2013: A priori testing of sparse adaptive polynomial chaos expansions using an ocean general circulation model database. Comput. Geosci., 17, 899-911, https://doi.org/10.1007/s10596-013-9361-3.

Xie, L., H. Liu, B. Liu, and S. Bao, 2011: A numerical study of the effect of hurricane wind asymmetry on storm surge and inundation. Ocean Modell., 36, 71-79, https://doi.org/10.1016/ j.ocemod.2010.10.001.

Xiu, D., and G. E. Karniadakis, 2002: The Wiener-Askey polynomial chaos for stochastic differential equations. SIAM J. Sci. Comput., 24, 619-644, https://doi.org/10.1137/S1064827501387826.
Zhang, F., and J. A. Sippel, 2009: Effects of moist convection on hurricane predictability. J. Atmos. Sci., 66, 1944-1961, https:// doi.org/10.1175/2009JAS2824.1.

Zhu, P., Y. Wang, S. S. Chen, M. Curcic, and C. Gao, 2016: Impact of storm-induced cooling of sea surface temperature on large turbulent eddies and vertical turbulent transport in the atmospheric boundary layer of Hurricane Isaac. J. Geophys. Res. Oceans, 121, 861-876, https://doi.org/10.1002/2015JC011320.

Zou, X., Z. Qin, and Y. Zheng, 2015: Improved tropical storm forecasts with GOES-13/15 imager radiance assimilation and asymmetric vortex initialization in HWRF. Mon. Wea. Rev., 143, 2485-2505, https://doi.org/10.1175/MWR-D-14-00223.1. 